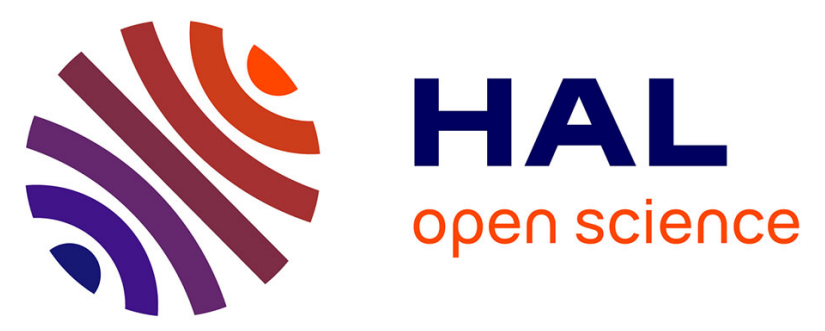

\title{
High-resolution observations and modeling of turbulence sources, structures, and intensities in the upper mesosphere
}

\author{
David C Fritts, Ling Wang, Gerd Baumgarten, Amber Miller, Marvin A \\ Geller, Glenn Jones, Michele Limon, Daniel Chapman, Joy Didier, Carl B \\ Kjellstrand, et al.
}

\section{To cite this version:}

David C Fritts, Ling Wang, Gerd Baumgarten, Amber Miller, Marvin A Geller, et al.. Highresolution observations and modeling of turbulence sources, structures, and intensities in the upper mesosphere. Journal of Atmospheric and Solar-Terrestrial Physics, 2017, 162, pp.57-78. 10.1016/j.jastp.2016.11.006 . hal-02091405

\section{HAL Id: hal-02091405 https://hal.science/hal-02091405}

Submitted on 5 Apr 2019

HAL is a multi-disciplinary open access archive for the deposit and dissemination of scientific research documents, whether they are published or not. The documents may come from teaching and research institutions in France or abroad, or from public or private research centers.
L'archive ouverte pluridisciplinaire HAL, est destinée au dépôt et à la diffusion de documents scientifiques de niveau recherche, publiés ou non, émanant des établissements d'enseignement et de recherche français ou étrangers, des laboratoires publics ou privés. 


\section{High-Resolution Observations and Modeling of Turbulence} Sources, Structures, and Intensities in the Upper Mesosphere

3

4

David C. Fritts ${ }^{1}$, Ling Wang ${ }^{1}$, Gerd Baumgarten ${ }^{2}$, Amber D. Miller ${ }^{3}$, Marvin A. Geller ${ }^{1}$, Glenn Jones ${ }^{3}$, Michele Limon ${ }^{3}$, Daniel Chapman ${ }^{3}$, Joy Didier ${ }^{3}$, Carl B. Kjellstrand ${ }^{3}$, Derek Araujo $^{3}$, Seth Hillbrand ${ }^{4}$, Andrei Korotkov ${ }^{5}$, Gregory Tucker ${ }^{5}$, and Jerry Vinokurov ${ }^{5}$

${ }^{1}$ GATS Inc., Boulder Division, Boulder, CO

${ }^{2}$ Leibniz Institute for Atmospheric Physics, Kühlungsborn, DE

${ }^{3}$ Department of Physics, Columbia University, New York, NY

${ }^{4}$ Department of Physics and Astronomy, California State University, Sacramento, CA

${ }^{5}$ Department of Physics, Brown University, Providence, RI

(1)

(1)

(1)

5

\author{
Corresponding author: D. C. Fritts
}

Address: GATS Inc., 3360 Mitchell Lane, Boulder, CO 80301

Email: dave@gats-inc.com

Phone: $720-274-4747$

9

.

5


Abstract

New capabilities for imaging small-scale instabilities and turbulence and for modeling gravity wave (GW), instability, and turbulence dynamics at high Reynolds numbers are

29 employed to identify the major instabilities and quantify turbulence intensities near the summer mesopause. High-resolution imaging of polar mesospheric clouds (PMCs) reveal a range of 31 instability dynamics and turbulence sources that have their roots in multi-scale GW dynamics at 32 larger spatial scales. Direct numerical simulations (DNS) of these dynamics exhibit a range of 33 instability types that closely resemble instabilities and turbulence seen in PMC imaging and by 34 ground-based and in-situ instruments at all times and altitudes. The DNS also exhibit the 35 development of "sheet-and-layer" (S\&L) structures in the horizontal wind and thermal stability 36 fields that resemble observed flows near the mesopause and at lower altitudes.

37 Both observations and modeling suggest major roles for GW breaking, Kelvin-Helmholtz 38 instabilities (KHI), and intrusions in turbulence generation and energy dissipation. Of these, 39 larger-scale GW breaking and KHI play the major roles in energetic flows leading to strong 40 turbulence. GW propagation and breaking can span several S\&L features and induce KHI 41 ranging from $\mathrm{GW}$ to turbulence scales. Intrusions make comparable contributions to turbulence 42 generation as instabilities become weaker and more intermittent. Turbulence intensities are 43 highly variable in the vertical and typically span 3 or more decades. DNS results that closely 44 resemble observed flows suggest a range of mechanical energy dissipation rates of $\varepsilon \sim 10^{-3}-10$ $45 \mathrm{Wkg}^{-1}$ that is consistent with the range of in-situ measurements at $\sim 80-90 \mathrm{~km}$ in summer.

47 Key Words: polar mesospheric clouds, gravity waves and instabilities, turbulence, MLT 48 dynamics 


\section{Introduction}

Turbulence has been recognized to be a ubiquitous component of the motion spectrum

54 throughout the atmosphere for more than five decades (e.g., Panofsky, 1959; Blamont and

55 DeJager, 1961; Obukhov, 1962; Witt, 1962; Lumley and Panofsky, 1964; Rees et al., 1972;

56 Woodman and Guillen, 1974; Zimmerman et al., 1977; Lilly, 1983; Wyngaard, 1992, 2010;

57 Gibson, 1996; and references therein). At all altitudes below the turbopause at $\sim 105-110 \mathrm{~km}$,

58 above which kinematic viscosity and thermal diffusivity largely suppress turbulence sources,

59 turbulence is believed to play multiple roles. In the mesosphere and lower thermosphere (MLT),

60 direct turbulence effects include 1) heating due to turbulence dissipation (Hocking, 1985;

61 Lübken, 1997; Becker and Schmitz, 2002; Lübken et al., 2002) and 2) mixing and transports of

62 heat, momentum, and constituents (e.g., Weinstock, 1978a, b; Schoeberl and Strobel, 1983; Chao

63 and Schoeberl, 1983; Fritts and Alexander, 2003, hereafter FA03; Bishop et al., 2004; Rapp et

64 al., 2004; Hecht et al., 2004). Importantly, however, the efficiency of turbulent transports

65 remains uncertain and strongly debated (Fritts and Dunkerton, 1985; Coy and Fritts, 1988;

66 Strobel et al., 1987; McIntyre, 1989; Becker and Schmitz, 2002; Becker and McLandress, 2009).

67 Turbulence also contributes indirectly to 3) the dissipation of, and heat and momentum

68 deposition by, gravity waves (GWs) and larger-scale motions (Hodges, 1967; Lindzen, 1981;

69 Walterscheid, 1981; Holton, 1982; Garcia and Solomon, 1987; FA03; Medvedev and Klaassen,

70 2003; Yigit, 2015) and 4) the generation of GWs accompanying larger-scale inhomogeneities in

71 turbulence layers (Dohan and Sutherland, 2005).

72 The importance of turbulence in the MLT derives from the various motions that

73 contribute to local instabilities at these altitudes. The large majority of the wind shear variance in

74 the MLT is due to propagation of GWs, tides, and planetary waves (PWs) from sources at lower

75 altitudes, and to their superpositions and interactions in the MLT accompanying their amplitude

76 increases with altitude. Of these motions, GWs contribute the major energy and momentum

77 fluxes because of their much larger vertical group velocities (FA03). GW interactions with the

78 larger-scale motions, and other GWs, cause refraction to smaller vertical scales, amplitude 
increases, instabilities, and dissipation, resulting in only $\sim 0.1 \%$ of the GW energy generated in

80 the troposphere reaching altitudes of $\sim 80-90 \mathrm{~km}$ (Fritts and VanZandt, 1993, hereafter FV93).

81 Despite this severe dissipation, GW velocity variances are $\sim 2$ decades larger near the mesopause 82 than near the tropopause (Balsley and Carter, 1982; Balsley and Garello, 1985) and mechanical 83 energy dissipation rates in the mesosphere, typically $\varepsilon \sim 10^{-3}-1 \mathrm{Wkg}^{-1}$, are similarly $\sim 2$ decades 84 larger than in the troposphere and lower stratosphere under stable conditions (Lawrence and 85 Balsley, 2013; Schneider et al., 2014; Fritts et al., 2016, hereafter F16).

86 High-resolution observations at multiple altitudes suggest that the dynamics accounting 87 for systematic GW energy dissipation, and energy and momentum deposition, often involve 88 "sheet-and-layer" (S\&L) structures comprising thinner, strongly stratified and sheared sheets and 89 thicker, weakly stratified and sheared layers (e.g., Gossard et al. 1985; Dalaudier et al. 1994; 90 Coulman et al. 1995; Luce et al. 1995, 1999; Balsley et al. 1998, 2003, 2012; Muschinski and 91 Wode 1998; Nastrom and Eaton 2001; Fritts et al., 2004, hereafter F04; Rapp et al., 2004; Chuda 92 et al. 2007; Clayson and Kantha, 2008). High-resolution direct numerical simulations (DNS) 93 reveal that such S\&L structures arise naturally from superposed and interacting GWs and larger94 scale winds having various spatial and temporal scales (Fritts et al., 2013, 2016; Fritts and Wang, 95 2013; hereafter F13, F16, and FW13, respectively). Both observations and DNS modeling reveal 96 that S\&L structures tend to exhibit the strongest potential temperature and horizontal wind 97 gradients, $d \theta / d z$ and $d u / d z$, up to $\sim 10$ times the mean gradients or larger, during the most active 98 instabilities and strongest turbulence. These decay with time in the absence of continued energy 99 inputs, but they can nevertheless extend for 10's of buoyancy periods (FW13; F16). Though 100 studied independently, these same dynamics also play major roles in oceans and lakes (e.g., 101 Osborn and Cox, 1972; Gregg, 1975; Thorpe, 1977, 2005; Osborn, 1980; Dillon, 1982; Wesson 102 and Gregg, 1994; Moum, 1996; Ferron et al., 1998; Gargett, 1999).

103 Recent DNS modeling has suggested that the "multi-scale dynamics" (MSD) that drive 104 S\&L structures and evolutions comprise superposed and interacting GWs and larger-scale flows, 105 sporadic instabilities of several types, and sporadic turbulence events (F13; F16). The major 
instabilities accompanying MSD include GW breaking, Kelvin-Helmholtz instabilities (KHI), and intrusions, and these can have very different influences on S\&L evolutions.

GW breaking within MSD occurs on scales comparable to and deeper than local S\&L scales, and leads to turbulence and mixing largely within the weakly-stratified layers. GWs also enhance the potential for KHI, both in the absence of, and accompanying, breaking. Hence, GW breaking and KHI often occur together or in close proximity. However, KHI occurs only on more strongly stratified and sheared sheets because the shears that enable KHI can only be sustained in high stratification. Sufficiently strong KHI can also lead to splitting of the initial sheet, as observed by Woods and Wiley (1972) in the ocean and modeled earlier with highresolution DNS or large-eddy simulation (e.g., Werne and Fritts, 1999; Fritts et al., 2012; hereafter WF99 and F12, respectively). When the MSD flows are energetic, e.g., a buoyancy Reynolds number $R e_{b}=\varepsilon / v N_{0}^{2}>20$ (Smyth and Moum, 2000), where $v$ and $N_{0}$ are kinematic viscosity and the mean buoyancy frequency, GW breaking and KHI predominate and the nearly laminar sheets have strong stratification and shear. As MSD become less energetic, intrusions compete with smaller-scale GW breaking, turbulence events are weaker, and the underlying GWs no longer contribute the strong shearing needed to maintain strongly stratified and sheared sheets (F16).

While considerable observational evidence for MSD has come from the lower atmosphere and oceans, multiple stratospheric and MLT observations have also revealed apparent S\&L structures and/or instability dynamics that are suggestive of MSD. Evidence of layering and multi-scale spatial and temporal variability in temperatures, winds, tracers, and/or turbulence intensities includes the following:

1) high-resolution balloon observations (Barat, 1982; Dalaudier et al., 1994; Gavrilov et al., 2005; Clayson and Kantha, 2008; Schneider et al., 2014),

2) MF, VHF, and UHF radar measurements of radar backscatter and/or spectral widths in the stratosphere and mesosphere (Woodman and Guillen, 1974; Sato and Woodman, 1982; Gage and Balsley, 1984; Hocking, 1985; Luce et al., 1995, 2006), and 
3) in-situ rocket and falling-sphere measurements (Lübken, 1997; Lübken et al., 2002; Rapp et al., 2004; Goldberg et al., 2006; Lehmacher et al., 2011; Szewczyk et al., 2013).

Additional evidence of instability dynamics suggestive of MSD influences and/or specific instability forms seen in airglow, polar mesospheric clouds (PMCs, also referred to as noctilucent clouds when viewed from below), chemical release imaging, and/or new DNS modeling includes the following:

4) localized GW enhancements, breaking, and/or instabilities (Yamada et al., 2001; Fritts et al., 2002, 2014a, hereafter F14a; Bossert et al., 2015),

5) localized KHI, several at peak GW vertical displacements, or along their descending phases (Witt, 1962; Fritts and Rastogi, 1985; Hecht, 2004; Hecht et al., 2005, 2014; Lehmacher et al., 2007; Pfrommer et al., 2009; Baumgarten and Fritts, 2014, hereafter BF14; Fritts et al., 2014b, c, hereafter F14b and F14c),

6) potential deep convective rolls (Larsen, 2000; Larsen et al., 2004), and

7) apparent intrusions of turbulent air into quiescent air seen in high-resolution PMC imaging (Miller et al., 2015, hereafter M15).

Thus, there is significant evidence for fairly ubiquitous MSD and S\&L features at all altitudes from the surface to $\sim 100 \mathrm{~km}$. This is not a universal characterization of atmospheric or MLT instability dynamics, however, as there is also evidence of more idealized instability dynamics accompanying large-amplitude, nearly-monochromatic or superposed GWs having vertical wavelengths larger than S\&L vertical scales. Importantly, larger vertical wavelengths and intrinsic phase speeds may allow such GWs to contribute to MSD at lower altitudes and amplitudes, and also attain larger amplitudes and rapid instability evolution without strong influences by smaller-scale S\&L features at higher altitudes. Examples include the following:

1) idealized GW and KHI dynamics predicted by theory and singular vector analysis and identified by DNS, such as GW breaking fronts, streamwise-aligned vortices, vortex rings, and $\mathrm{KHI}$ secondary instabilities observed in PMC and airglow imaging to date 
(e.g., Achatz, 2005, 2007; F09a; F09b; Fruman and Achatz 2012; F13; Remmler et al. 2013; BF14; F14b; F14c; Fruman et al., 2014; Hecht et al., 2014; M15), and

2) nonlinear dynamics comprising various wave-wave and wave/mean-flow interactions, including "self-acceleration" (SA) dynamics, modulational instabilities, and overturning of localized GW packets at large amplitudes and/or intrinsic frequencies (Sutherland,

165

166

167 2001, 2006a, 2006b; Doser and Sutherland, 2011; Fritts et al., 2015, hereafter F15), Summarizing, various analysis and modeling methodologies are now providing important insights into the various instability dynamics contributing to GW dissipation and turbulence production throughout the atmosphere. DNS, in particular, is identifying specific instability features at finite amplitude that resemble those observed by high-resolution imaging in the MLT. Our goals in this paper are to explore the ability of high-resolution DNS to describe realistic GW and MSD instability and turbulence events and aid in the interpretation of observed events in the MLT. To achieve these goals, our efforts will include three components:

1) employ ground-based and balloon-borne PMC imaging to identify the types, scales, and stages of instability dynamics accounting for MLT turbulence,

2) use these results to select and scale DNS of idealized GW breaking and MSD energetics that best approximate the observed events, and

3) employ the DNS results to assess the implied turbulence intensities and variability.

Overviews of PMC observations from the ground and from a stratospheric balloon over Antarctica are presented in Section 2. DNS methods and results employed for comparisons with these observations are described in Sections 3 and 4. Applications of the DNS results to the interpretation of PMC observations are discussed in Sections 5 and 6. These results are employed to estimate the magnitudes and variability of $\varepsilon$ for the dynamics observed in the PMC fields in Section 7. A discussion of these results in relation to previous studies is provided in Section 8 , and our summary and conclusions are presented in Section 9.

\section{Observations of MLT Dynamics in PMCs}

\subsection{PMC Ground-Based Imaging in Norway}



$\left(63.4^{\circ} \mathrm{N}\right)$ on 1 August 2009. A Canon 50D with a $135-\mathrm{mm}$ lens provided continuous imaging at a cadence of $\sim 0.5 \mathrm{~s}$ in a narrow field-of-view (FOV) of $\sim 28 \times 41 \mathrm{~km}$ (horizontal and range) at a central elevation angle of $\sim 21^{\circ}$ and a horizontal range of $\sim 250 \mathrm{~km}$ yielding zonal and meridional resolution of $\sim 10$ and $20 \mathrm{~m}$. A Canon 450D with a 24-mm lenses provided wide FOV imaging of

191 the PMC layer over an area of $\sim 600 \times 600 \mathrm{~km}$ at a cadence of $\sim 30 \mathrm{~s}$.

\section{2.2. Lidar Measurements of PMC in Norway}

193 The Rayleigh lidar at the Arctic Lidar Observatory for Middle Atmosphere Research 194 (ALOMAR) in northern Norway $\left(69.3^{\circ} \mathrm{N}\right)$ supports multiple measurement modes (see, e.g., von 195 Zahn et al., 2000; Baumgarten et al., 2012; Kaifler et al., 2013). For our purposes, it employed a 196 lidar single-shot acquisition (LISA) capability to measure PMC backscatter and vertical 197 displacements at range and time resolutions of $25 \mathrm{~m}$ and $0.033 \mathrm{~s}$. The implications of these 198 measurement will be discussed below.

199 2.3. PMC Observations from the Southern Hemisphere Stratosphere

$200 \quad$ PMC imaging was obtained serendipitously by star cameras for the E and B Experiment 201 (EBEX), which measured the polarization of the cosmic microwave background from a 202 stratospheric balloon over Antarctica from 29 December 2012 to 9 January 2013 (M15). Star 203 camera field-of-views (FOVs) of $4.1 \times 2.7^{\circ}$ from an altitude of $\sim 35 \mathrm{~km}$ at zenith angles of $\sim 39$ and $20443^{\circ}$ yielded $\sim 4.4 \times 3.9-\mathrm{km}$ projected FOVs at the altitude of maximum PMC brightness, $\sim 82 \mathrm{~km}, \mathrm{a}$ 205 range of $\sim 62 \mathrm{~km}$, and a central image separation of $\sim 5.8 \mathrm{~km}$. The narrow star camera FOVs 206 yielded spatial resolution of $\sim 2.2 \mathrm{~m}$, thus resolving the inner scale of turbulence, $207 l_{0}=9.9\left(v^{3} / \epsilon\right)^{1 / 4} \sim 10 \mathrm{~m}$, for $v \sim 1 \mathrm{~m}^{2} \mathrm{~s}^{-1}$ and $\epsilon \sim 1 \mathrm{~m}^{2} \mathrm{~s}^{-3}$ at $82 \mathrm{~km}$ (see comparisons with rocket 208 measurements below), when apparent PMC advection was small. The close alignment of the two 209 star camera FOVs, and the imaging cadence of $450 \mathrm{~ms}$, provided multiple cases in which the 210 same dynamics in the same or closely-spaced regions were imaged 2 or more times. During the 211 11-day experiment, the star cameras collected $\sim 40,000$ images, of which $\sim 50 \%$ provided usable 212 images for purposes of assessing small-scale instability and turbulence dynamics. 


\section{$213 \quad 3 . \quad$ DNS Models and Simulation Parameters}

214 As noted above, we expect many types of instabilities to lead to turbulence in the MLT.

215 Of these, GWs having vertical wavelengths $\lambda_{z} \sim 2 \mathrm{~km}$ or larger and relatively high intrinsic

216 frequencies, $\omega_{i}>>f$, where $f$ is the inertial frequency, readily lead to breaking via either $2 \mathrm{D}$

217 and/or 3D instabilities, depending on the environment and the relevant Reynolds number, 218 defined as $R e=\lambda_{z}^{2} / v T_{b}$ with buoyancy period $T_{b}=2 \pi / N_{0}$ (Fritts et al., 2009a, b, hereafter F09a, 219 F09b),. At much smaller $\omega_{i}$, e.g., within a factor of $\sim 2-3$ of $f$, the dominant instabilities at 220 Richardson numbers $R i=N^{2} /(d U / d z)^{2}=N^{2} h^{2} / U^{2}<1 / 4$ and $R i<0$ are KHI (Lelong and Dunkerton, 221 1998a, b; Thorpe, 1999). GW distortions and intensification of local sheets of high stability and 222 wind shear also lead to KHI that may be highly localized (e.g., Hecht et al., 2005, 2014; F13, 223 F14b, F14c, F16). However, F14b found very weak turbulence to occur for $R i=0.2$ and $R e=2500$, 224 while flows for $R i=0.05$ and $R e=1000$ become 3D but remain laminar. Thus, the latter are a very 225 conservative threshold for turbulence generation and these yield $h=15(v / N)^{1 / 2}$. Taking $v \sim 1 \mathrm{~m}^{2} \mathrm{~s}^{-1}$ 226 and $N \sim 0.03 \mathrm{~s}^{-1}$ for a weakly stratified sheet (as opposed to a layer having $N \sim 0.01 \mathrm{~s}^{-1}$ or less), we 227 obtain a threshold wavelength $\lambda_{x T} \sim 4 \pi \sim 1.1 \mathrm{~km}$ below which KHI remain laminar. KHI at 228 smaller $R i$ and larger $R e$ have already been studied via DNS and high-resolution observations 229 (e.g., F14b, F14c), and will not be considered here. Importantly, those studies suggested an often 230 significantly larger effective turbulent viscosity, $v_{t u r b}$, that could further decrease $R e$ and $\lambda_{x T}$ $231 v_{\text {turb }}{ }^{1 / 2}$ by $\sim 3$ times or more for new instabilities. The other major instabilities occurring in 232 observed flows and simulated MSD are intrusions (see M15 and F16). These do not appear to

233 provide direct evidence of the spatial scales of the MSD, however they do distinguish MSD from 234 more idealized flows. Given this, our focus here will be on modeling of GW breaking and MSD 235 for comparisons with observations.

236 3.1. Equations

237 Our applications of DNS of GW breaking and MSD to MLT turbulence employ our 238 previous results scaled to MLT events and turbulence intensities (e.g., F09a; F09b; F13; F16).

239 These involve solutions of the 3D, Boussinesq, nonlinear Navier-Stokes equations, which are 
nondimensionalized with respect to a primary GW vertical wavelength $\lambda_{z}$, a uniform buoyancy

241 period $T_{b}$, and the velocity scale $U_{0}=\lambda_{z} / T_{b}$. The resulting equations may be written as

$$
\partial \boldsymbol{u} / \partial t+\boldsymbol{u} \cdot \nabla \boldsymbol{u}=-\nabla p+R i \theta \mathbf{z}+R e^{-1} \nabla^{2} \boldsymbol{u}
$$

$\partial \theta / \partial t+\boldsymbol{u} \cdot \nabla \theta=(\operatorname{Pr} R)^{-1} \nabla^{2} \theta$

Here $\boldsymbol{u}=(u, v, w), p$, and $\theta$ are the total velocity vector, pressure, and potential temperature, $N^{2}=\left(g / \theta_{0}\right) \mathrm{d} \theta_{0} / \mathrm{d} z=g \beta / \theta_{0}, g, \theta_{0}$, and $\beta$ are gravity, mean $\theta$ and its gradient, and $\mathbf{z}$ is a unit vector in the vertical. As previously, $R e=U_{0} \lambda_{z} / v=\lambda_{z}{ }^{2} / v T_{b}, R i=N^{2} \lambda_{z}{ }^{2} / U^{2},=4 \pi^{2}$, and the Prandtl number, $\operatorname{Pr}=v / \kappa=1$ (where $\kappa$ is thermal diffusivity), is assumed for computational efficiency (e.g., F09a).

The linear inviscid dispersion relation arising from Eqs. (1-3) is given by

$$
m^{2}=\left(k^{2}+l^{2}\right)\left(N^{2} / \omega_{i}^{2}-1\right)
$$

252 propagation, $m$ is the vertical wavenumber, $\omega_{i}=k_{h} c$ is the GW intrinsic frequency (assuming zero 253 large-scale mean wind), $k_{h}^{2}=k^{2}+l^{2}, c$ is the GW horizontal phase speed, and we assume mean

254 wind shear and curvature to be negligible. The primary GW wavenumber vector for each DNS is $\boldsymbol{k}=(k, l, m)=\left(2 \pi / \lambda_{x}, 0,-2 \pi / \lambda_{z}\right)$ in geographic coordinates. A tilted computational domain is employed

256 for both GW breaking and MSD applications (see Fig. 1), yielding a primary GW wavenumber of $\boldsymbol{k}^{\prime}=\left(k^{\prime}, l^{\prime}, m^{\prime}\right)=(0,0,-1)$, which allows a much more compact computational domain for $m / k>>1$.

Following Andreassen et al. (1998), we employ the negative eigenvalue $\left(\lambda_{2}\right)$ of the tensor

$259 \boldsymbol{L}=\boldsymbol{\Omega}^{2}+\boldsymbol{S}^{2}$, where $\boldsymbol{\Omega}$ and $\boldsymbol{S}$ are the rotation and strain tensors, to identify flow features having 260 strong rotational character (Jeong and Hussain, 1995). Such features have large negative $\lambda_{2}$, 261 whereas pure shearing motions yield $\lambda_{2}=0$. Thus $\lambda_{2}$ allows us to follow the transition from initial 262 instability structures, through vortex interactions and instabilities, to fully developed turbulence 263 and decay. We also compute the mechanical energy dissipation rate, defined as $\varepsilon=2 R e^{-1}<S_{i j} S_{i j}>$, 264 to provide estimates of $\varepsilon$ for these various dynamics for the observed MLT event scales.

\section{3.2. GW Breaking Configuration}


We employ previous DNS of GW breaking (F09a; F09b) for initial GW amplitudes

$267 a=u^{\prime} / c_{i}=0.9$ and 1.1 (for intrinsic phase speed $c_{i}=(c-U)$ and mean wind $U$ along $c$ ), an intrinsic 268 frequency $\omega_{i}=N / 3.2 \quad\left(T_{G \mathrm{~W}}=3.2 T_{b}\right)$, and $R e=\lambda_{z}{ }^{2} / v T_{b}=10,000$. The computational domain has 269 dimensions $\left(X^{\prime}, Y^{\prime}, Z^{\prime}\right)=(3.4,2.22,1) \lambda$, where $\lambda=2 \pi /|\boldsymbol{k}|$ is the GW wavelength (see Fig. 1a). The 270 two cases require comparable spectral resolution, with as many as $\left(N_{x}, N_{y}, N_{z}\right)=(2400,1600,800)$ 271 Fourier modes employed at the times of strongest small-scale turbulence. The underlying 272 dynamics are described in detail by F09a and F09b. Applications to instability forms and 273 turbulence structures observed in PMCs are described in Section 4.

\section{3.3. MSD Configuration}

MSD employed here are those described by F16 at $R e=100,000$, which achieve $R e_{b} \sim 20$ -

27630 during the strongest turbulence, and scaled to the MLT. As in F16, initial conditions include a 277 convectively stable GW and a stable small-scale shear flow. The GW has an amplitude $a=0.5$, $278 \omega_{i}=N / 10\left(T_{G \mathrm{~W}}=10 T_{b}\right)$, wavenumber $\left(k^{\prime}, l^{\prime}, m^{\prime}\right)=(0,0,-1)$, and exhibits a minimum $R i \sim 4$ where $279 d \theta^{\prime} / d z \sim 0$ and $\mathrm{d} u^{\prime} / \mathrm{d} z \sim N / 2$. The initial mean motion is given by a sinusoidal streamwise velocity 280 having 5 cycles across the vertical projection of the GW $\lambda$ having $(d U / d z)_{\max }=2 N$ such that the 281 minimum mean shear is $R i=1 / 4$. The streamwise and spanwise domain extents are $282 X^{\prime}=Z^{\prime} /(5 \tan \phi)=1.999 Z^{\prime}$ and $Y^{\prime}=0.5 \lambda_{z}=0.4975 Z^{\prime}$ (see Fig. 1b). The highest model resolution used 283 for this DNS is $(4320,1080,2160)$ Fourier modes. These MSD, and their implications for 284 instability and turbulence structures observed in PMCs, are described in Sections 4 and 5.

\section{3.4. Simulation of PMC images}

286 The instability dynamics examined here occur on timescales of only a few minutes. Over 287 such short intervals, PMC brightness can be approximated as a passive tracer (G. Thomas, PC, 288 2016: Chandran et al., 2016). Hence, we parameterize PMC brightness as a function of the initial $289 \theta_{0}$, which is monotonically increasing and nearly conserved following the air motion, as

$$
B(z)=B_{0} \exp \left[-\left(z-z_{P M C}\right)^{2} / 2 \sigma_{z}^{2}\right]=B_{0} \exp \left[-\left(\theta_{0}-\theta_{P M C}\right)^{2} / 2 \sigma_{\theta}^{2}\right]
$$

291 where $z_{P M C}$ and $\theta_{P M C}$ are PMC altitude and $\theta_{0}$ at the peak of PMC brightness and $\sigma_{z}=$ $292 z_{F W H M} / 2(2 \ln 2)^{1 / 2}$ corresponds to an PMC layer having a typical full width-half maximum 
293 (FWHM) brightness of $z_{F W H M}$. Examples of Rayleigh lidar observations at ALOMAR described

294 above are shown for reference in Fig. 2. These reveal that the PMC brightness layer can have $295 z_{F W H M}$ as small as $\sim 50-100 \mathrm{~m}$, and perhaps less, accompanying strong layering in the presence of 296 energetic small-scale GW and instability dynamics. Additional evidence for such thin layers 297 accompany observed and DNS MSD flows that yield $d \theta / d z$ and $N^{2}$ maxima up to 10 times mean 298 values or larger and high $\operatorname{Re}$ (F16, and references therein). This is a particularly useful feature of 299 PMC layers because it provides a potential to observe structures occurring at very small scales.

\section{4. DNS of Idealized GW Breaking and MSD}

\section{4.1. DNS of GW breaking, instabilities, and turbulence} Floquet theory employed by F09a suggested initial GW instabilities comprising streamwise-aligned (streamwise vorticity, spanwise wavenumber) counter-rotating rolls. The parallel DNS show these to be approximately horizontal initially, and to evolve to finiteamplitude and induce intensifying vortex sheets above (below) having negative (positive) streamwise vorticity (Andreassen et al., 1998). These dynamics are illustrated in Figs. 3 and 4 with volumetric views of $\lambda_{2}$ at $1 T_{b}$ intervals beginning before strong breaking for GW amplitudes $a=0.9$ and 1.1 and other parameters specified above. Note that we have displayed these fields with GW propagation upward and to the right in order to have the same orientation as in the ground-based PMC imaging discussed below.

311 Where these vortices dip below the coldest phase of the GW (above the maxima of $u$ ' and $312 w^{\prime}$ ), they interact with the spanwise vortex sheets above the most unstable (smallest $N^{2}$ ) phase of

313 the GW. The downward motions induce stretching and intensification of the opposite spanwise 314 vortex sheets below, and these structures link to form a succession of vortex rings along the GW 315 phase that expand in time and advect upward at the maxima of $u^{\prime}$ and $w^{\prime}$ along $c$, though at a 316 velocity smaller than $c$ (Figs. 3 and 4; panels a, b, e, and f). The resulting vortex rings yield 317 plunging motions downward and in the direction of GW propagation (Figs. 3 and 4; panels $\mathrm{f}$ and 318 g). They also cause coherent linkages between adjacent GW fronts that act to organize the 319 transitional instability structures that cannot be seen in the $\lambda_{2}$ volumetric views in Figs. 3 and 4 . 
320 Thereafter, strong interactions among adjacent vortices induce "twist-wave" perturbations of the

321 various vortex structures that cause their fragmentation and rapid collapse to smaller scales 322 comprising the turbulence cascade (Figs. 3 and 4; panels b-d and f-h; F09b; Andreassen et al., 323 1998; Fritts et al., 1998). The evolutions from coherent vortex rings to strong turbulence span $324 \sim 1 T_{b}$ or less, or a few minutes for a typical mean $N$, with a more rapid evolution at a larger 325 amplitude and/or higher $\omega_{i}$ (new paper in preparation).

326 4.2. DNS of MSD, instabilities, and turbulence

327 As noted above, instabilities exhibit many forms in MSD flows throughout their 328 evolutions. These arise as a result of interactions among the larger-scale mean and GW motions 329 that largely account for the S\&L structures and the instability and turbulence dynamics induced 330 within them. Examples of the S\&L structures and instabilities occurring at several stages of an 331 MSD DNS described by F16 are shown in the $N^{2}(x, z)$ and $\log _{10} \varepsilon(x, z)$ fields at left and right in 332 Fig. 5. Color scales in both cases are from weak (blue) to strong (red), $N^{2}$ varies by more than a 333 decade, and $\varepsilon$ varies by more than 4 decades at each time. Strong GW breaking and large-scale 334 KHI arise at early stages following S\&L formation when large GW amplitudes and shears 335 provide significant energy sources for instabilities and turbulence (e.g., Fig. 5, panels a-d and g$336 \mathrm{j})$. At later stages, instabilities are weaker and more intermittent, turbulence is weaker and more 337 confined vertically, and intrusions (e.g., Fig. 5, panels e, f, k, and l) play a competitive role with 338 weaker GW breaking events.

339 Finally, we note that both the idealized GW breaking and MSD dynamics are described 340 in a Boussinesq DNS having specified initial conditions. As such, they are "spin-down" DNS 341 without continuous energy inputs by GWs propagating from below. This does not limit the 342 ability to compare instability dynamics and/or turbulence intensities with atmospheric 343 observations at low or high altitudes (e.g., F13, F14b, F16) at sufficiently high Re. But the earlier 344 times in each DNS having more energetic GWs, instabilities, and turbulence are more 345 representative of atmospheric conditions in which there are significant GW fluxes of energy to 346 higher altitudes. In contrast, the later times in each DNS more closely approximate times where 
347 GW vertical energy fluxes are relatively weak. The DNS of idealized GW breaking and MSD

348 described here are employed below to interpret and quantify instability dynamics and turbulence 349 intensities observed in PMCs from the ground and a stratospheric balloon. The former provides 350 continuous viewing of dynamics that may span multiple $T_{b}$; the latter does not track the 351 evolutions of specific dynamics, but does provide valuable insights into small-scale instability 352 and turbulence dynamics that are impossible to obtain at any other altitude in the atmosphere.

\section{5. GW Breaking Dynamics Observed in Ground-Based PMC Imaging}

We first examine an apparent GW breaking event observed over northern Norway on 1 August 2009. These dynamics occurred following an extensive display of KHI beginning 22:30 UT, having constant phases aligned roughly northwest to southeast (NW-SE), advecting towards the southwest $\left(\sim 40^{\circ} \mathrm{W}\right.$ of $\left.\mathrm{S}\right)$ at $\sim 70 \mathrm{~ms}^{-1}$, and exhibiting very slow evolutions in time (e.g., individual $\mathrm{KH}$ billow events spanning $\sim 10$ min or longer). This display includes the KHI Ri 0.20 event described in detail by Baumgarten and Fritts (2014) and F14b. Throughout, the KHI 360 structures exhibited significant modulations in phase and amplitude (see Fig. 6a), suggesting a 361 complex shear environment likely reflecting spatial variations accompanying small-scale GWs. radar at ALOMAR, $\sim 450 \mathrm{~km} \mathrm{~N}$ of the observations from 22 to $24 \mathrm{UT}$. These had 2-km and 1-hr resolution, but appeared to be consistent with observed PMC advection at $\sim 82 \mathrm{~km}$ and our expectation of mean winds towards the SW decreasing with altitude during summer.

Beginning at $\sim 23: 10$, larger-scale PMC brightness variations became apparent that

367 suggest GW modulations of the wind and temperature fields and the PMC layer on larger spatial 368 scales. Extended KHI structures persisted in the presence of the larger-scale GWs at several 369 locations beyond 23:20 (see Fig. 6). Despite more complex KHI structures at small spatial 370 scales, their evolution timescales did not change appreciably. However, the wind shears enabling 371 KHI apparently diminished with time, and evidence of KHI disappeared thereafter (e.g., see the 372 outer edges of the PMC images at 23:30 and thereafter in Fig. 6). 
The GW field contributing to the changing instability environment had several apparent

374 components: 1) one having a horizontal wavelength $\lambda \sim 70 \mathrm{~km}$ and propagating slowly towards 375 the southeast (SE) throughout, 2) a second having $\lambda \sim 30 \mathrm{~km}$ and propagating more rapidly 376 towards the north-northeast (NNE) from 23:30-50, and 3) a third localized GW having $\lambda \sim 50 \mathrm{~km}$, 377 a large amplitude from $\sim 23: 30-50$, and propagating towards the south-southeast (SSE). These 378 GW phases and propagation directions are shown with the white, red, and pink lines and arrows 379 in Fig. 6b-d, respectively. Of these, the large-amplitude, smaller-scale GW propagating towards 380 the SSE appears to have had the largest influences on instability forms and alignments in the 381 region highlighted by the yellow circles in Fig. 6. Hence, we will assume this GW was the major 382 cause of the instabilities seen to occur after 23:30 in Fig. 6, though the others likely also 383 contributed to the local shear and stability fields. Shown with a green trapezoid in Fig. 6c is the 384 region in which we will focus our discussion of the instability dynamics during this event.

385 The dominant GW initiated a series of bright bands aligned nearly along its direction of 386 propagation at two phases at 23:30 and 23:40 (see the features within the yellow circles in Fig. $3876 \mathrm{~b}$ and $6 \mathrm{c})$. These features are very similar to those previously associated with GW breaking seen 388 in PMC (Fritts et al., 1993), and seen prior to the formation of vortex rings in the DNS shown in 389 Figs. 3 and 4. In this case, however, the two primary GW phases were neither linear nor parallel 390 due to the influences of other GWs also present. Initial streamwise-aligned features were more 391 distinct at 23:30 prior to the occurrence of instabilities between these phases. Changing GW 392 superpositions and induced instabilities in this region thereafter extended beyond 24:00.

393 By 23:40, small-scale instabilities having 3D character appeared in the lee of the leading 394 GW phase at lower right (to the NW). These initial dynamics are shown from 23:42-45 UT at 1395 min intervals in Fig. 7a-d. The volume viewed is shown with the green trapezoid in Fig. 6c and 396 the viewing angle for these images is from ahead and to the right of the primary GW propagation 397 direction, and below the horizontal plane of the PMC by $\sim 21^{\circ}$. The images in Fig. $7 \mathrm{a}$ and $7 \mathrm{~d}$ are 398 also shown mapped and viewed from above to show their true spatial scales in Fig 7e and 7f. As 399 in the DNS, the initial bands assigned the scales of the subsequent 3D instability features seen in 
their lee. Additionally, the time scales of the instability dynamics changed dramatically between 23:30 and 23:40. Whereas KHI observed prior to $\sim 23: 40$ evolved over several $T_{b}$, the apparent GW breaking dynamics shown in Fig. 7 evolved significantly over $3 \min \left(\sim 0.6 T_{b}\right)$.

The images at top reveal complex structures that we identify as vortex rings that appear to be overlapping because of the shallow imaging slant view. In reality, the vortices are likely displaced horizontally rather than vertically within the GW field, as they occur at only one phase of the DNS of GW breaking seen in Figs. 3 and 4. Their alignments appear to have been at successive positions along the plane of GW propagation (lower left to upper right in Fig. 7a-d), with apparently overlapping vortex rings staggered at adjacent horizontal locations normal to the plane of GW propagation, as seen in the DNS, especially Fig. 3. Their diameters at these times were as large as $\sim 8-10 \mathrm{~km}$, and likely $\sim 20-50 \%$ larger than when they first formed at smaller amplitudes, based on the expansions seen in the DNS results. Our DNS reveal that vortex ring spacing along the plane of GW propagation, and between vortex rings occurring on adjacent

413 vortex sheets, varies with GW amplitude and intrinsic frequency, and likely with the background

414 wind shear and orientation, however. In particular, new DNS reveal that vortex ring spacings and 415 evolution timescales decrease with increasing $\omega_{i}$ (L. Wang, pers. comm., 2016).

416 The three initial vortex rings at the more southward locations (upper and lower portions 417 of the yellow ovals in Fig. 7a and 7e, respectively) exhibit bright trailing vortices extending 418 generally upward and to the west from their interiors (as in Figs. 3b and 4b) due to the viewing 419 geometry. Especially significant in this sequence is the rapid decay of the coherent vortex rings 420 over its 3 -min $\left(\sim 0.6 T_{b}\right)$ duration that is consistent with their decay time scale seen in the DNS 421 results in Figs. 3 and 4. This is dramatically faster than the evolutions of the earlier KHI at the 422 PMC altitude and is a clear distinction between $\mathrm{KHI}$ and GW breaking dynamics. Additional, but 423 less distinct, features are also seen to the west and north (left and below in Fig. 7a-d) of those 424 highlighted, and they likewise decay over the same interval.

425 We explore these comparisons further using Eq. (5) to compute PMC brightness fields for 426 the GW breaking events shown in Figs. 3 and 4 at four times with a PMC layer thickness of 
$z_{F W H M}=0.1 \lambda_{z}$. The results for $a=0.9$ from $21-22 T_{b}$ and $a=1.1$ from 10.2-11.2 $T_{b}$ at $0.33 T_{b}$

428 intervals are shown viewed from below at left and right in Fig. 8, respectively. In each case, the 429 PMC layer was placed to coincide with the vortex rings in the most unstable phase of the GW.

430 Both cases exhibit trailing vortices, as seen in the PMC images, and a rapid evolution from 431 coherent vortex rings to turbulent motions over $\sim 1 T_{b}$. That with $a=0.9$ more clearly illustrates 432 vortex ring expansion; that with $a=1.1$ exhibits a more rapid vortex ring breakdown to turbulence 433 and a smaller vortex ring spacing (given periodic boundaries, Fig. 11d).

434 The subsequent evolution of this region shown from 23:47-50 UT in Fig. 9a-d reveals the 435 further breakdown of the vortex rings discussed above (see yellow ovals). These images also 436 suggest the development of new vortex rings at even larger scales (see the brightening curved 437 features at the right edges of Fig. 8e and 8f), but these are seen primarily in the wide FOV, as 438 they advect out of the narrow FOV to the SW over the next $\sim 5 \mathrm{~min}$.

\section{$439 \quad$ 6. GW Breaking Dynamics and MSD Observed in Stratospheric PMC Imaging}

Miller et al. (2015) reported an initial exploration of serendipitous PMC imaging from a

441 stratospheric balloon in the interpretation of dynamics near $82 \mathrm{~km}$. Here, we perform additional 442 comparisons of stratospheric imaging of PMCs and DNS modeling to quantify the character, 443 scales, and energetics of key instability dynamics arising due to GW breaking and MSD at PMC 444 altitudes. This process enables estimates of the contributions of these dynamics to the energy 445 dissipation rate $\varepsilon$ in the MLT presented in Section 7 below.

Many PMC images obtained by the EBEX stratospheric balloon experiment include

447 features that appear to be evidence of either relatively monochromatic GW breaking, smaller448 scale KHI, or more complex MSD events (Miller et al., 2015). These can often be identified by 449 PMC brightness variations arising due to advection and deformation of the PMC layer by larger450 and smaller-scale GWs, their various instability forms, and turbulence structures. The unique 451 aspect of these observations is the extremely high spatial resolution that can be achieved in cases 452 where the PMC layer is very thin accompanying the underlying dynamics, e.g., resolving 453 features having spatial scales as small as $\sim 10-20 \mathrm{~m}$. 


\subsection{GW breaking dynamics observed in stratospheric PMC imaging}

Two examples that appear to be at relatively early stages in the evolution of a breaking $456 \mathrm{GW}$ are shown in Fig. 10a and 10b. These do not correspond to the same event, as EBEX 457 imaging only occasionally, and accidentally, acquired images of the same PMC field spaced by 45810 's of seconds or more. Nevertheless, the two images appear to capture features that we 459 recognize as slightly different stages of breaking GW. That in Fig. 10a closely resembles a 460 simulated PMC (Fig. 10c) suggesting the overturning "front" for the GW breaking case with $461 a=1.1$ and $z_{F W H M}=100 \mathrm{~m}$ at a time just after that in Fig. 4b. That in Fig. 10b suggests a strange 462 diagonal pattern that likewise resembles a simulated PMC (Fig. 10d) for the GW breaking case 463 with $a=0.9$ and $z_{F W H M}=100 \mathrm{~m}$ at a time near that in Fig. 3b. These images indicate that there are 464 occasions when the larger-scale MLT dynamics (i.e., vertical scales larger than the S\&L scales in 465 MSD) are characterized locally by approximately monochromatic GW breaking rather than less 466 energetic MSD. The apparent overturning front reveals approximately streamwise vortices at the 467 initial stages of instability that may allow an estimate of the depth of the layer that is unstable, 468 based on the spanwise (along the front) spacing of the major vortex features. Comparing the 469 characteristic spacing with the DNS suggests a vertical wavelength of $\lambda_{z} \sim 2-4 \mathrm{~km}$, but provides 470 little guidance on $\lambda_{h}$ or $\omega_{i}$. The diagonal patterns in Fig. $10 \mathrm{~b}$ and $10 \mathrm{~d}$ suggest a $\lambda_{h}>5 \mathrm{~km}$, given 471 that the true PMC image does not capture the full streamwise GW phase structure.

More quantitative assessments are possible in cases where specific instability dynamics 473 directly related to intrinsic GW parameters are observed. The clearest examples of such at larger 474 spatial scales identified to date appear to be vortex rings, as simulated in the GW breaking DNS 475 shown in Figs. 3 and 4, and as seen in initial EBEX PMC imaging (Miller et al., 2015). Images 476 of apparent vortex rings in three different events are shown in Fig. 11a-c. That in Fig. 11a shows 477 the full image having a horizontal FOV of 4.4x3.9 km and reveals a well-developed vortex ring 478 diameter of $\sim 4 \mathrm{~km}$ and a spacing between rings of $\sim 4 \mathrm{~km}$. The diameter is indicative of a GW $479 \lambda_{z} \sim 8-10 \mathrm{~km}$, and the close spacing is suggestive of $\omega_{i} \sim N / 3$ or greater. Additionally, the apparent 480 bright trailing vortices extending to the lower right of the vortex rings suggest vortex ring self- 
481 advection and GW propagation towards the upper left. A simulated PMC from the DNS shown

482 in Fig. 11d for a GW propagating upward (as seen from below) is shown for comparison in Fig.

483 11d. This captures the darker vortex rings, their successive displacements, and an indication of 484 brighter trailing vortex features, suggesting agreement of the underlying dynamics.

Fig. $11 \mathrm{~b}$ shows another apparent vortex ring that was propagating largely away from the viewing platform (downward in the PMC image), based on the orientation of the bright trailing 487 vortices. This vortex ring had a diameter of $\sim 1.5-2 \mathrm{~km}$ and appeared to be undergoing 488 breakdown (as seen in Figs. 3c and 4c), or to have occurred in an already turbulent region. It had 489 no other vortex rings in close proximity, suggesting an $\omega_{i}$ comparable to, or lower than, shown in 490 Figs. 3 and 4, based on DNS observations of closer vortex ring spacing at $\omega_{i} \sim N / 2$ to N/1.4.

$491 \quad$ Fig. 11c shows a PMC image that reveals an apparent succession of vortex rings that had 492 similar character, were apparently linked by trailing vortices, and were self-advecting towards 493 the right, suggesting GW propagation in this direction. These features are strikingly similar to a 494 simulated PMC using the DNS for $a=1.1$ at $\sim 10.5 T_{b}$, a short time following the image in Fig. $4 \mathrm{~b}$.

495 The diameters of these structures are up to $\sim 1 \mathrm{~km}$ and they appear to be closely spaced along the 496 GW propagation direction, suggesting a GW $\lambda_{z} \sim 2 \mathrm{~km}$ and $\omega_{i} \sim N / 3$ or larger in this case.

\section{6.2. MSD observed in stratospheric PMC imaging}

498 EBEX stratospheric PMC imaging has also yielded multiple examples that confirm DNS 499 predictions of various MSD flows, several of which are described below. Fig. 12a shows what 500 appear to have been cusp-like structures having longitudinal scales of up to $\sim 1 \mathrm{~km}$, lateral scales 501 of $\sim 100-300 \mathrm{~m}$, some organization of these features in lateral rows, and apparent strong shearing 502 of the lower edges towards the lower left (along the arrow).

503 The simulated PMC image in Fig. 12b was obtained using the method described in 504 Section 3.4 with $z_{F W H M}=200 \mathrm{~m}$ at the bottom edge of the region of local $\mathrm{GW}$ breaking having 505 large $\varepsilon$ shown in the black oval in Fig. 5h. Inspection of the corresponding $N^{2}$ field in Fig. 5b 506 reveals this to be a sheet having elevated stability, and the corresponding velocity field (not 507 shown) confirms a significant wind shear with increasing winds at lower altitudes along the 
508 arrow. As in Fig. 12a, cusp-like features seen in Fig. 12b exhibit apparent shearing consistent

509 with the wind direction below, some lateral alignments, and lateral scales of $\sim 5-10 \%$ of the MSD

510 domain width $\left(\sim \lambda_{z} / 2\right)$. Finally, we note that the cusp-like features in Fig. 12 have many

511 similarities to the vortex rings identified at larger spatial scales, including ring-like highlights

512 and streamwise vortices extending from their centers a multiple sites in the observed and

513 simulated fields. Thus, they could be manifestations of small-scale GW breaking in a more

514 chaotic environment.

515 Equating the cusp scales between the EBEX and MSD simulated PMC images suggests

516 an MSD overall depth of $\sim 6 \mathrm{~km}$. This scale is less important in this case than the S\&L spatial

517 scales that constrain this event. This depth is likely $\sim 1 \mathrm{~km}$ in this region of the MSD flow, based

518 on the DNS shown in Fig. 5. Hence, we expect the MSD DNS to provide a reasonable 519 approximation of the associated $\varepsilon$ for these specific dynamics in the discussion below.

520 Other instabilities that are seen in a number of stratospheric PMC images are intrusions.

521 Two examples are shown in Fig. 13a and 13b. Their apparent directions of motion are shown

522 with arrows and the image widths are $\sim 3$ and $4.4 \mathrm{~km}$, respectively. These observations confirm

523 the occurrence and importance of these dynamics predicted by the MSD DNS, at least in cases

524 where other dynamics that lead to stronger turbulence are not present (F13, F16). An intrusion

525 occurring in the MSD DNS at late times is shown evolving from a laminar to a turbulent flow in

526 Fig. 5k and 51. Simulated PMC responses assuming a PMC $z_{F W H M}=100$ and $300 \mathrm{~m}$ in the region

527 within the black oval in Fig. 51 are shown in Fig. 13c and $13 \mathrm{~d}$ to illustrate the sensitivity of the

528 PMC response to the PMC layer depth. These reveal that thinner layers provide greater 529 sensitivity to smaller-scale dynamics, as argued above. Unlike GW breaking, there is no current 530 way to evaluate $\varepsilon$ for intrusions without a means of assessing their scales. This may change in 531 cases where their depths, relative motions, and/or turbulence spectra can be directly assessed.

\section{7. Energy Dissipation Rates in the MLT}

533 The discussion above identified four events observed in PMC imaging from the ground

534 and stratosphere that compare reasonably with DNS of GW breaking for idealized and MSD 
535 flows and enable some quantification of GW parameters. These allow DNS results to be scaled

536 to the observed dynamics that enable estimates of $\varepsilon$ for the observed events at PMC altitudes.

537 The cases for which this can be done with some confidence include the following:

538 Case 1: GW breaking over Norway (Fig. 7) exhibiting vortex rings having initial diameters of $\sim 7 \mathrm{~km}$ or larger suggesting a GW having $a>1, \lambda_{z} \sim 15 \mathrm{~km}$ or larger, and $\omega_{i}>N / 3$, nondimensional DNS to the observed dynamics and inferred spatial scales. For both idealized 548 GW breaking and MSD, the scaling factor is $S=\lambda_{z}{ }^{2} / T_{b}{ }^{3} . S$ will vary by $\sim 2$ decades because of the 549 large range of $\lambda_{z} \sim 2-15+\mathrm{km}$ inferred in our discussions of the events above. $S$ will also vary with $550 \mathrm{~N}$, which is determined by temperature fields on larger scales than the $\mathrm{GW}$ breaking event, i.e., 551 mean, tidal, planetary wave, and larger-scale GWs, that can be measured by ground-based and 552 in-situ instruments. Where these are unknown, uncertainties could be as large as $\sim 2$ in the large553 scale $N$ based on in-situ measurements to date. We have no direct measurements of $N$ for any of 554 the PMC events discussed here. However, the mean PMC environment at $\sim 82 \mathrm{~km}$ over 555 ALOMAR has $T \sim 150 \mathrm{~K}, N \sim 0.023 \mathrm{~s}^{-1}$, and $T_{b} \sim 270 \mathrm{~s}$ (Rapp et al., 2004). Below, we examine the 556 implications of each of the above cases for MLT instability event scales and turbulence 557 intensities, assuming these values for our analyses. Event parameters are listed in Table 1.

558 7.1. Case 1: GW breaking with $\lambda_{z} \sim 15+\mathrm{km}$ The GW breaking event observed over Norway revealed large-scale vortex rings 560 implying a GW having $a>1, \lambda_{z} \sim 15 \mathrm{~km}$ or larger, and $\omega_{i}>N / 3$. Together, these parameters imply 
561 that the GW breaking DNS for $a=1.1$ shown in Fig. 4 represents a conservative estimate of the 562 instability and turbulence intensities for this case, perhaps by a significant factor. Horizontal distributions of nondimensional $\varepsilon$ at the times of largest mean $\langle\varepsilon>$ (where 564 brackets denote a domain average) for $a=0.9$ and 1.1 (i.e., Figs. 3d, 3h, 4d, and 4h) viewed from 565 below and the side (with GW propagation up and to the right) are shown in Fig. 14a, 14b, 14e,

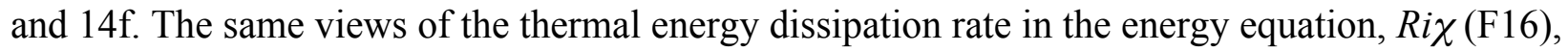
567 are shown in Fig. 14c, 14d, 14g, and 14h. These reveal that despite strong vortex interactions, 568 turbulence generation, and mixing along the most unstable phase of the GW spanning several $T_{b}$,

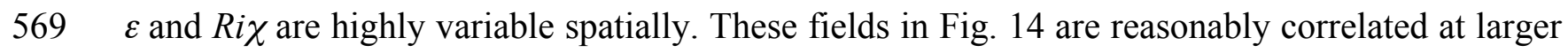
570 spatial scales, but their maxima often are not. In particular, there are many cases in which $\varepsilon$ and


572 strong mixing has driven the strong thermal gradients to the edges of the mixing regions. Probability distribution functions (PDFs) of $\log _{10} \varepsilon$ for $a=0.9$ at 21, 22, 24, 30, and $40 T_{b}$ 574 are shown in Fig. 15a. Those for $a=1.1$ at 9.2, 10.2, 12.2, 20, and $30 T_{b}$ are shown in Fig. $15 \mathrm{~b}$. 575 The two earliest times in each case are during the onset of turbulence at larger spatial scales. As 576 the initial vortex rings evolve, the PDFs acquire approximately log-normal distributions, and 577 these persist to very late times. The strongest turbulence in each case occurs $\sim 2 T_{b}$ after vortex 578 ring formation, and $\left\langle\varepsilon>\right.$ and $\varepsilon_{\text {high }}$ (above which the highest $1 \%$ of $\varepsilon$ occur) are shown with 579 vertical lines in Fig. 15a and 15b. These are $\sim 0.7-1.1$ decades larger than at the times of initial 580 coherent vortex rings for $a=0.9$ and 1.1 , respectively. Hence, we expect maximum turbulence to 581 occur $\sim 8-10$ min after the appearance of coherent vortex rings (and large-scale $u^{\prime}, w^{\prime}$, and $T^{\prime}$ for

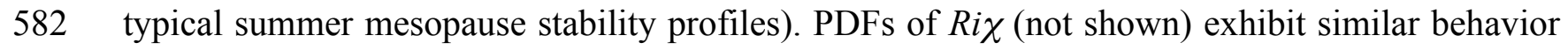
583 following the onset of instabilities and turbulence (e.g., F09b). Because of the broad, 584 approximately $\log$-normal distributions of $\varepsilon$ and $\chi$ accompanying turbulence (they span $\sim 4$ 585 decades at the times of strongest turbulence), only $\sim 15-20 \%$ of the values are above their domain 586 means. Even for the vertical and spanwise means shown in Fig. 14 (which have narrower 
distributions spanning only $\sim 2$ decades, see the color bars in Fig 14; also see the local PDFs in

588 Fig. 8 of FW13), the largest values are strongly localized at the sites of the previous vortex rings.

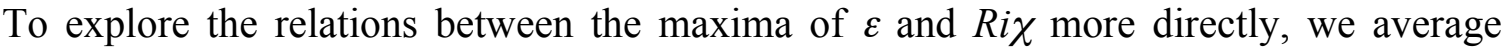
these quantities over 64 adjacent points to provide high confidence in these values, yielding values at $\sim 10^{8}$ locations. As above, this reduces the range of values, but these still span $>3$ decades. Scatter plots of these values for the larger $0.1-0.01 \%$ (black dots) and $0.01-0.001 \%$


594 16b, respectively.



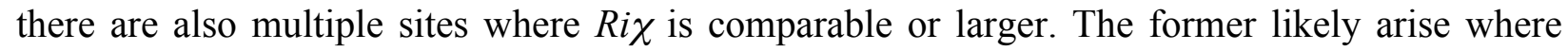
597 strong mixing of the thermal gradients has already occurred, the latter likely are from regions within the strongly stratified and sheared sheets within which turbulence events first arise and strong thermal and velocity gradients are still entwined. Distributions for the largest Ri $\chi$ exhibit different behavior. And there is almost no overlap between the largest $0.001 \%$ of $\varepsilon$ and Ri , at most 4 locations in $\sim 10^{8}$. The largest Rix typically accompany $\varepsilon$ values between $\sim 1$ and $10 \%$ of 602 the maximum, thus apparently do not have much smaller associated $\varepsilon$. This is because smallscale velocity gradients (and significant $\varepsilon$ ) are required to maintain strong thermal gradients.

Turning to implications of these dynamics for turbulence intensities, we dimensionalize the PDFs for $a=0.9$ and 1.1 by $S=\lambda_{z}^{2} / T_{b}^{3}=11 \mathrm{Wkg}^{-1}$ (or $\mathrm{m}^{2} \mathrm{~s}^{-3}$ ), yielding maxima of $<\varepsilon>\sim 0.22$ and $0.55 \mathrm{Wkg}^{-1}$ and $\varepsilon_{\text {high }} \sim 2.4$ and $6 \mathrm{Wkg}^{-1}$ for these cases, given the separations between their

607 locations shown with vertical lines in Fig. 15a and 15b. The mean values $<\varepsilon>$ are comparable to, 608 or larger than, the largest estimates by in-situ probes at PMC altitudes of $\sim 0.3 \mathrm{Wkg}^{-1}$ (e.g., 609 Strelnikov et al., 2009). The implied $\varepsilon_{\text {high }}$ are much larger than have been observed at these 610 altitudes, especially as the inferred GW parameters suggest that the likely $<\varepsilon>$ and $\varepsilon_{\text {high }}$ are larger 611 than those for $a=1.1$.

There are several explanations that appear plausible. Very large $<_{\varepsilon}>$ and $\varepsilon_{\text {high }}$ likely 613 accompany very strong events that are infrequent and most often highly localized spatially and 
614 temporally; see, e.g., the strong spatial localization implied by the broader PMC fields shown in

615 Fig. 6 and the transience (i.e., a duration of $\sim 1-2 T_{b}$ ) of strong maxima implied by Figs. 14b, 14f,

$61615 \mathrm{a}$, and 15b. Hence, the likelihood that any single rocket measurement would observe such an

617 event at the location and time of maximum $\langle\varepsilon>$ is very small, perhaps less than $0.1 \%$. This

618 fraction would increase significantly, of course, where ground-based measurements were able to

619 provide real-time identification of specific events and expected times of maximum turbulence.

620 The likelihood of observing $<\varepsilon>$ and/or $\varepsilon_{\text {high }}$ even sampling such an event is also small because

621 of the limited portions of the GW and turbulence field that have large $\left\langle\varepsilon>\right.$ and/or $\varepsilon_{\text {high }}$ along a

622 single near-vertical rocket trajectory implied by the horizontal $\varepsilon$ fields in Fig. 14a and $14 \mathrm{~b}$.

623 Indeed, sampling a turbulence field having high intermittency would require multiple vertical

624 profiles to develop confidence that a representative mean value was likely to be obtained.

Another reason that sampling $\langle\varepsilon\rangle$ and/or $\varepsilon_{\text {high }}$ using current in-situ instruments is challenging is that they measure temperature rather than velocity fluctuations, hence estimate the

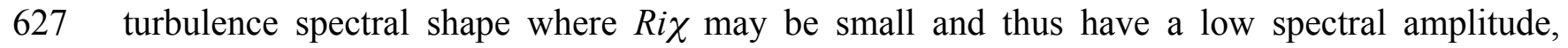

628 despite large $\varepsilon$ (see Fig. 16a). Finally, smaller measured than predicted $\varepsilon_{\text {high }}$ may arise because 629 in-situ measurements typically average over $\sim 30-100 \mathrm{~m}$ along the rocket trajectory, whereas $\varepsilon_{\text {high }}$ 630 occur accompanying the strongest small-scale vortices (and largest velocity shears) near the 631 turbulence inner scale, $l_{0}=9.9\left(v^{3} / \varepsilon\right)^{1 / 4} \sim 10 \mathrm{~m}$ or less (Lübken, 1997), accompanying the strongest 632 events. Several of these influences can be evaluated by employing current in-situ measurement 633 methods to sample our various DNS data, and we expect to perform such studies in the future.

634 7.2. Case 2: GW breaking with $\lambda_{z} \sim 8-10 \mathrm{~km}$

635 The vortex rings shown in Fig. 11a have diameters of $\sim 4 \mathrm{~km}$ and appear to have been 636 observed prior to further instabilities (and ring expansion), implying GW breaking for $a \sim 1, \lambda_{z} \sim 8$ $63710 \mathrm{~km}$, and $\omega_{i} \sim N / 3$. These parameters yield $S \sim 3-5 \mathrm{Wkg}^{-1}$, hence maximum $<\varepsilon>\sim 0.06-0.1$ and $6380.15-0.25 \mathrm{Wkg}^{-1}$ and maximum $\varepsilon_{h i g h} \sim 0.7-1.1$ and 1.6-2.6 $\mathrm{Wkg}^{-1}$ for the two cases shown in Figs. 6393 and 4 . These $<\varepsilon>$ values are comparable to the larger values measured at the PMC altitude, i.e., $640<\varepsilon>\sim 0.1-0.3 \mathrm{Wkg}^{-1}$ (e.g., Rapp et al., 2004; Strelnikov et al., 2009). These magnitudes suggest 
641 that such GW and instability scales may be somewhat more representative of those occurring

642 more frequently at $\sim 82 \mathrm{~km}$.

\section{7.3. Case 3: $G W$ breaking with $\lambda_{z} \sim 2 \mathrm{~km}$}

644 The smaller vortex rings shown in Fig. 11c having maximum diameters of $\sim 1 \mathrm{~km}$ imply $645 \mathrm{GW}$ breaking for $a \sim 1, \lambda_{z} \sim 2 \mathrm{~km}$, and $\omega_{i}>N / 3$, hence $S \sim 0.2 \mathrm{Wkg}^{-1}$ or somewhat larger. These 646 imply much weaker turbulence than suggested by the larger-scale vortex rings in Cases 1 and 2. 647 Scaling in this case implies $<\varepsilon>\sim 0.004$ and $0.01 \mathrm{Wkg}^{-1}$ and $\varepsilon_{\text {high }} \sim 0.04$ and $0.1 \mathrm{Wkg}^{-1}$ for $a=0.9$ 648 and 1.1, with larger values if $\omega_{i}$ exceeds $N / 3$ by a significant amount. These and smaller 649 turbulence magnitudes should be expected for GW breaking events at quite small horizontal and 650 vertical scales, such as might be implied by $\sim 2-\mathrm{km}$ vertical scales in lidar or in-situ $T^{\prime}(z)$ profiles 651 obtained with very little temporal averaging. Similar $\lambda_{z}$, but $\lambda_{h} \sim 10-30 \mathrm{~km}$ or larger, imply 652 smaller $<\varepsilon>$ and $\varepsilon_{\text {high }}$ because of the smaller GW vertical group velocities of such motions.

653 7.4. Case 4: MSD with $\lambda_{z} \sim 6 \mathrm{~km}$

654 The cusp patterns seen in Fig. 12a suggest GW breaking similar to that in a shallow layer 655 of the MSD DNS shown in Fig. 5h assuming a domain depth of $6 \mathrm{~km}$. PDFs of $\varepsilon$ for this event 656 are shown at $5,10,12,22$, and $32 T_{b}$ in Fig. 15c. For reference, time series of domain mean $[\varepsilon]$ 657 are compared for the two GW breaking events and the MSD event described above in Fig. 15d. 658 That for the MSD DNS is shown at 5x amplitude for comparison with the GW breaking DNS.

From the $\varepsilon$ field shown in Fig. 5h, we see that the cusp-like features occur in a region that 660 spans nearly the full range of $\varepsilon$ in the full domain at the time of the cusp-like event. The inferred 661 domain depth implies $S \sim 2 \mathrm{Wkg}^{-1}$, thus a maximum $<\varepsilon>\sim 0.016 \mathrm{Wkg}^{-1}$ at the time of the cusp 662 occurrence, and $\varepsilon_{\text {high }} \sim 0.25 \mathrm{Wkg}^{-1}$.

\section{8. Discussion}

664 Our study has focused on identification of GW instability dynamics observed in PMC 665 imaging, their spatial and/or temporal scales, and the turbulence intensities, $<\varepsilon>$ and $\varepsilon_{\text {high }}$, 666 anticipated via comparisons with high-resolution DNS of these dynamics. To achieve results 667 with the highest confidence, we have focused on dynamics that were well defined in our various 
668 PMC imaging, thus on events that exhibited the largest instability scales that could be identified.

669 Because turbulence intensities vary as $\lambda_{z}{ }^{2}$ for any event, our studies likely emphasized the 670 stronger turbulence sources during these observations due to their more easily identified and 671 quantified dynamics. Hence, the $\left\langle\varepsilon>\right.$ and $\varepsilon_{h i g h}$ accompanying the most energetic phases of these 672 events are surely significantly larger than mean values at these altitudes. As an example, our range of estimates of $\left\langle\varepsilon>\sim 0.06-0.55 \mathrm{Wkg}^{-1}\right.$ at the most intense phases 674 of the stronger events analyzed in detail mostly exceed the values observed in a PMC 675 environment at $\sim 82 \mathrm{~km}$ of $\sim 0.001-0.3 \mathrm{Wkg}^{-1}$ inferred in previous radar and in-situ assessments 676 by up to 20 times (e.g., Lübken, 1997; Lübken et al., 2002; Rapp et al., 2004; Engler et al., 677 2005; Latteck et al., 2005; Strelnikov et al., 2009; Szewczyk et al., 2013) and elsewhere at the 678 same altitudes (e.g., Hocking, 1988). This is especially true given that we regard $\left\langle\varepsilon>\right.$ and $\varepsilon_{h i g h}$ 679 estimated using the GW breaking DNS for $a=1.1, \lambda_{z} \sim 15 \mathrm{~km}$, and $\omega_{i}=N / 3.2$ to be quite 680 conservative for Case 1 with estimated GW parameters of $a>1, \lambda_{z} \sim 15 \mathrm{~km}$ or larger, and $\omega_{i}>N / 3$. 681 The estimate of $\varepsilon_{h i g h} \sim 6 \mathrm{Wkg}^{-1}$ for this case far exceeds all estimates at $\sim 82 \mathrm{~km}$ and is comparable 682 to the largest magnitudes $\left(\sim 1-10 \mathrm{Wkg}^{-1}\right)$ reported near the mesopause in the papers cited above. 683 The largest $\varepsilon$ in Fig. 15 are another $\sim 3$ times higher.

Possibly more interesting, but to which PMC estimates can contribute only peripherally, 685 are the larger and more continuous estimates of $\langle\varepsilon>$ as atmospheric stability increases approaching and above the polar summer mesopause. In-situ measurements at these altitudes

687 have revealed relatively continuous turbulence intensities increasing from PMC altitudes to $688<\varepsilon>\sim 0.03-1 \mathrm{Wkg}^{-1}$ above $\sim 85 \mathrm{~km}$, with peak values as high as $<\varepsilon>\sim 2-10 \mathrm{Wkg}^{-1}$ in each profile, 689 and often occurring just above the mesopause (Rapp et al., 2004; Szewczyk et al., 2013). These 690 measurements suggest that strong turbulence near the mesopause is apparently not intermittent, 691 but more nearly continuous in space and time, despite the potential for under-estimates of true $692<\varepsilon>$ magnitudes noted above.

693 Several aspects of MLT GW dynamics contribute to sustained, strong turbulence near 694 and above the polar summer mesopause. There is a ubiquitous spectrum of GWs having various 
695 (and evolving) intrinsic parameters $\left(a, \lambda_{z}, \lambda_{h}, \omega_{i}\right.$, direction of propagation) that account for

696 continuous fluxes of energy and momentum into the polar summer MLT from sources primarily

697 at lower altitudes (e.g., Balsley and Garello, 1985; FV93; FA03). There are also environmental

698 influences that cause GWs to increase in relative amplitude and lead to instabilities and

699 turbulence throughout the atmosphere. These include the following:

700 1) continuous increases in GW amplitudes with increasing altitude due to decreasing density,

7012 2) successive instability cycles at similar altitudes for GWs having large $\omega_{i}$ and $\lambda_{z}$ due to only partial GW attenuation accompanying breaking (F09a, F09b),

3) successive instabilities at higher altitudes for large GWs with $\omega_{i}$ and $\lambda_{z}$ due to only partial GW amplitude reductions accompanying breaking at lower altitudes,

4) wind shears due to mean, tidal, planetary wave, and larger-scale GWs that can induce decreasing $\lambda_{z}$ and increasing $a$ for GWs experiencing decreasing $\omega_{i}$,

5) increasing $N^{2}$ that drives decreasing $\lambda_{z}$, increasing $u^{\prime} / w^{\prime} \sim \lambda_{h} / \lambda_{z}$ and $a$, hence increasing GW instabilities (VanZandt and Fritts, 1989, hereafter VF89; FV93), and

713 turbulence dynamics, but these are often localized within the GW field and their detection

714 depends on their amplitudes and the spatial resolution of the imager. Large-scale instabilities

715 (horizontal scales up to $10 \mathrm{~km}$ or larger) that are easily detected can occupy from a few $\%$ to a

716 majority of a large FOV. However, strong, local instabilities that evolve rapidly are often closely

717 associated with the largest-amplitude (brightest) GWs having $\lambda_{h} \sim 30-60 \mathrm{~km}$ or smaller. Strong

718 turbulence likely accompanies the stronger instabilities, whereas weaker turbulence is long-

719 lasting and thus assured and likely widespread, but very difficult to observe.

720 The various effects noted above are accounted for statistically in a spectral model of the 721 evolution and dissipation of GWs with altitude (and varying $N^{2}$ ) that is constrained by 
722 observations and theory. The model initially addressed only increases in $N^{2}$ (VF89), but was later

723 generalized to include realistic altitude variations throughout the atmosphere (FV93). This theory

724 yields an estimate of $<\varepsilon>\sim 0.28 \mathrm{Wkg}^{-1}$ near the mesopause without the enhanced contribution due 725 increasing $N$ (FV93); the enhanced $\left\langle\varepsilon>\right.$ (and $\varepsilon>\varepsilon_{\text {high }}$ ) may be significantly larger, depending on 726 the degree and depth of $N^{2}$ variations at the polar summer mesopause (VF89).

Finally, turbulence typically survives for long times following its initiation. As examples,

728 instabilities leading to turbulence have shear and buoyancy energy production terms that span

729 several $T_{b}(\mathrm{~F} 09 \mathrm{~b})$, and $<\varepsilon>$ decreases by only $\sim 3-4$ times over the final $10 T_{b}$ of the PDFs shown

730 in Fig. 14. While not a component of this study, KHI secondary instabilities and turbulence have 731 comparable time scales for their evolutions (WF99; F12, F14b). Thus, successive, or unrelated, 732 new GW or MSD instabilities have significant time over which to evolve prior to cessation of 733 turbulence due to previous events.

734 Significant unknowns related to these studies at present include the following:

735 1) definition of the local environmental $N$ needed to quantify estimates of $S$ and $\varepsilon$,

736 2) influences of background turbulence, and a smaller "turbulent" Reynolds number,

Evidence for the influences of background turbulence on KHI seen in PMCs and $\mathrm{OH}$ airglow

741 was provided in the studies by Baumgarten and Fritts (2014), F14b, Hecht et al. (2014), and

742 F14c. In these cases, background $v_{\text {turb }}>v$ (and $R e_{\text {turb }}<R e$ ) were inferred due to larger observed 743 spatial scales of secondary instabilities than were anticipated by DNS of KHI in the absence of 744 an elevated viscosity. Estimates in these cases ranged from $v_{\text {turb }} \sim 5-40 v$ (and $R e_{\text {tur }} \sim 0.025-0.2$ $745 R e$ ), though much higher values are likely to accompany stronger turbulence sources. Assuming 746 the same influences occur for other types of instabilities, we should expect our DNS of GW 747 breaking and MSD to be appropriate for larger spatial scales that would otherwise have much 748 higher $R e$. As an example, the GW breaking assumed to cause the vortex rings in Case 1 had 
$749 R e=\lambda_{z}^{2} / v T_{b} \sim 8 \times 10^{5}$, assuming no turbulent viscosity. The Floquet theory for the DNS shown in

750 Figs. 3 and 4 (F09a) suggests that a higher Re does not likely change the instability character,

751 scales, or growth rates. However, larger $\varepsilon$ accompany larger $R e$ for the same underlying

752 dynamics (F16). Hence smaller $v_{t u r b}$ and larger $R e_{t u r b}$ would yield even larger $\varepsilon$ estimates than

753 discussed above.

754 Additional effects that accompany strong instabilities and turbulence in the MLT (and

755 throughout the atmosphere) include local heating and turbulent mixing and transport. Turbulence

756 heating rates are $\sim 100 \mathrm{~K} /$ day for $\varepsilon \sim 1 \mathrm{Wkg}^{-1}$ and thus comparable in magnitude to the adiabatic

757 cooling driven by GW-induced upwelling near the polar summer mesopause. Turbulence is also

758 anticipated by many to contribute a strong downward heat flux near and above the polar summer

759 mesopause, but modeling of these dynamics has yet to confirm (or contradict) these expectations.

\section{$7609 . \quad$ Summary and Conclusions}

761 We have employed high-resolution DNS to explore the implications of instability

762 features observed in high-resolution imaging of PMCs for turbulence sources and intensities near

763 the polar summer mesopause. Imaging by ground-based cameras in Norway and serendipitously

764 by star cameras as part of a cosmology experiment flown on a stratospheric balloon over

765 Antarctica yielded evidence of various instability types suggesting idealized and multi-scale GW

766 dynamics at PMC altitudes. Observations of instability features and scales enabled estimates of

767 the underlying GW intrinsic properties and amplitudes in four cases.

768 Specific results and implications of our study include the following:

769 1) PMC imaging reveals an active dynamical environment with larger-scale GWs always

770 present and smaller-scale instabilities occurring frequently, as also noted previously,

7712 2) the dominant instabilities include KHI, GW breaking, and intrusions at multiple scales,

772 3) vortex rings are a frequent instability form accompanying GW breaking at various scales,

773 4) vortex rings occurring at large scales with close spacing imply large GW amplitude $a$,

774 vertical wavelength $\lambda_{z}$, and intrinsic frequency $\omega_{i}$, and large corresponding energy and

775 momentum fluxes, 
795 its resolution.

\section{Acknowledgments}

797 Research described here was supported by NASA grants NNX16AF64G, NNX08AG40G, and 798 NNX07AP36H and by NSF grants AGS-1136269, AGS-1445783, AGS-1510354, and AGS799 1452183. This project has received funding from the European Union's Horizon 2020 research 800 and innovation program under grant 653980/ARISEII. The authors gratefully acknowledge 801 discussions of GW-driven PMC brightness variations with Amal Chandran and Gary Thomas 802 and the use of the Werne/NWRA Triple Code for the simulations presented within. We also 
803 acknowledge the DoD High Performance Computing Modernization Program for access to

804 supercomputer platforms that allowed the simulations reported here.

805

806

807

\begin{tabular}{|l|l|l|l|l|l|l|l|l|l|}
\hline Case & Event type & $\begin{array}{l}\lambda_{z} \\
(\mathbf{k m})\end{array}$ & $\boldsymbol{a}$ & $\begin{array}{l}\boldsymbol{\omega}_{\boldsymbol{0}} \\
(\boldsymbol{N})\end{array}$ & $\begin{array}{l}\lambda_{\boldsymbol{h}} \\
\mathbf{( k m )}\end{array}$ & $\mathbf{C}=\lambda_{z}{ }^{2} / \boldsymbol{T}_{\boldsymbol{b}}{ }^{3}$ & $\boldsymbol{R} \boldsymbol{e}=\lambda_{z}{ }^{2} / \boldsymbol{v} \boldsymbol{T}_{\boldsymbol{b}}$ & $\begin{array}{l}\boldsymbol{\varepsilon}_{\text {mean }} \\
\left(\mathbf{W k g}^{-1}\right)\end{array}$ & $\begin{array}{l}\boldsymbol{\varepsilon}_{\text {high }} \\
\left(\mathbf{W k g}^{-1}\right)\end{array}$ \\
\hline 1 & ideal. GWB & $>15$ & $>1$ & $>0.3$ & & 7.3 & $8.3 \times 10^{5}$ & $>0.55$ & $>6$ \\
\hline 2 & ideal. GWB & $8-10$ & $\sim 1$ & $>0.3$ & & $2.1-3.2$ & $2.4-3.7 \times 10^{5}$ & $\sim 0.06-0.25$ & $\sim 0.7-2.6$ \\
\hline 3 & ideal. GWB & 2 & $\sim 1$ & $>0.3$ & & 0.13 & 15,000 & $\sim 0.004-0.01$ & $\sim 0.04-0.1$ \\
\hline 4 & MSD GWB & 1 & $>1$ & & & 1.2 & $1.3 \times 10^{5}$ & 0.016 & 0.25 \\
\hline
\end{tabular}

808 Table 1. GW parameters and $\varepsilon$ estimates for idealized and MSD GW breaking (GWB) cases.

809

810

811 References

812 Achatz, U., 2005: On the role of optimal perturbations in the instability of monochromatic

813 gravity waves, Phys. of Fluids, 17, 094107, doi:10.1063/1.2046709.

814 Achatz, U., 2007: The primary nonlinear dynamics of modal and nonmodal perturbations of

815 monochromatic inertia-gravity waves, J. Atmos. Sci., 64, 74-95.

816 Andreassen, O., P. O. Hvidsten, D. C. Fritts, and S. Arendt, 1998: Vorticity dynamics in a

817 breaking gravity wave, 1. Initial instability evolution, J. Fluid Mech., 367, 27-46.

818 Balsley, B. B., and D. A. Carter, 1982: The spectrum of atmospheric velocity fluctuations at 8

819 and 86 km, Geophys. Res. Lett., 9, 465-468.

820 Balsley, B. B., R. G. Frehlich, M. L. Jensen, Y. Meillier, and A. Muschinski, 2003: Extreme

821 gradients in the nocturnal boundary layer: Structure, evolution, and potential causes. J.

$822 \quad$ Atmos. Sci., 60, 2496-2508. 
823 Balsley, B. B., and R. Garello, 1985: The kinetic energy density in the troposphere, stratosphere,

824 and mesosphere: A preliminary study using the Poker Flat MST radar in Alaska, Radio Sci., $82520(6), 1355-1361$.

826 Balsley, B. B., M. L. Jensen, and R. Frehlich, 1998: The Use of state-of-the-art kites for profiling 827 the lower atmosphere, Bound. Layer Meteorol., 87, 1-25.

828 Balsley, B. B., D. A. Lawrence, R. F. Woodman, and D. C. Fritts, 2012: Fine-scale 829 characteristics of temperature, wind, and turbulence in the lower atmosphere $(0-1,300 \mathrm{~m})$ 830 over the south Peruvian coast, Bound. Layer Meteorol., DOI 10.1007/s10546-012-9774-X.

831 Baumgarten, G., A. Chandran, J. Fiedler, P. Hoffmann, N. Kaifler, J. Lumpe, A. Merkel, C. E. 832 Randall, D. Rusch, and G. Thomas, 2012: On the horizontal and temporal structure of 833 noctilucent clouds as observed by satellite and lidar at ALOMAR (69N), Geophys. Res. Lett., 834 39, L01803, doi:10.1029/2011GL049935.

835 Baumgarten, G., and D. C. Fritts, 2014: Quantifying Kelvin-Helmholtz instability dynamics 836 observed in noctilucent clouds: 1. Methods and observations, J. Geophys. Res. Atmos., 119, 837 9324-9337, doi:10.1002/2014JD021832.

838 Chandran, A., D. W. Rusch, G. E. Thomas, S. E. Palo, G. Baumgarten, E. J. Jensen, and A. W. 839 Merkel, 2012: Atmospheric gravity wave effects on polar mesospheric clouds: A comparison 840 of numerical simulations from CARMA 2D with AIM observations, J. Geophys. Res., 117, 841 D20104,doi:10.1029/2012JD017794.

842 Chuda, T., R. Kimura, and H. Niino, 2007: Vertical Fine Structures of Temperature and Water 843 Vapor in the Free Atmosphere, J. Meteorol. Soc Japan, 85 (5), 583-597.

844 Clayson, C. A., and L. Kantha, 2008: On turbulence and mixing in the free atmosphere inferred 845 from high-resolution soundings, J. Atmos. Ocean. Tech., 25, 833-852, 846 DOI:10.1175/2007JTECHA992.1.

847 Coulman, C. E., J. Vernin, and A. Fuchs, 1995: Optical seeing mechanism of formation of thin 848 turbulent laminae in the atmosphere, Appl. Opt., 34, 5461-5474, 1995. 
849 Coy, L., and D. C. Fritts, 1988: Gravity wave heat fluxes: A Lagrangian approach, J. Atmos. $850 \quad$ Sci., $45,1770-1780$.

851 Dalaudier, F., C. Sidi, M. Crochet, and J. Vernin, 1994: Direct evidence of "sheets" in the 852 atmospheric temperature field, J. Atmos. Sci., 51, 237-248.

853 Dillon, T. M., 1982: Vertical overturns: A comparison of Thorpe and Ozmidov length scales. J. 854 Geophys. Res., 87, 9601-9613.

855 Dosser, H. V., and B. R. Sutherland, 2011: Weakly nonlinear non-Boussinesq internal gravity $856 \quad$ wavepackets, Physica D, 240, 346-356.

857 Engler, N., R. Latteck, B. Strelnikov, W. Singer, M. Rapp, 2005: Turbulent energy dissipation 858 rates observed by Doppler MST Radar and by rocket-borne instruments during the 859 MIDAS/MaCWAVE campaign 2002. Annales Geophysicae, European Geosciences Union, 23 (4), pp.1147-1156.

861 Ferron, B., H. Mercier, K. Speer, A. Gargett, and K. Polzin, 1998: Mixing in the Romanche 862 fracture zone. J. Phys. Oceanogr., 28, 1929-1945.

863 Fritts, D. C., and M. J. Alexander, 2003: Gravity dynamics and effects in the middle atmosphere, $864 \quad$ Rev. Geophys., 41, doi:10.1029/2001RG000106.

865 Fritts, D. C., S. Arendt, and O. Andreassen, 1998: Vorticity dynamics in a breaking internal 866 gravity wave, 2. Vortex interactions and transition to turbulence, J. Fluid Mech., 367, 47-65.

867 Fritts, D. C., G. Baumgarten, K. Wan, J. A. Werne, T. Lund, 2014b: Quantifying Kelvin868 Helmholtz Instability Dynamics Observed in Noctilucent Clouds: 2. Modeling and 869 Interpretation of Observations, J. Geophys. Res., 119 (15), 9324-9337.

870 Fritts, D. C., and T. J. Dunkerton, 1985: Fluxes of heat and constituents due to convectively 871 unstable gravity waves, J. Atmos. Sci., 42 , 549-556.

872 Fritts, D. C., J. R. Isler, G. E. Thomas, and Ø. Andreassen, 1993: Wave breaking signatures in 873 noctilucent clouds, Geophys. Res. Lett., 20, 2039-2042. 
874 Fritts, D. C., B. Laughman, T. S. Lund, and J. B. Snively, 2015: Self-acceleration and instability

875 of gravity wave packets: 1. Effects of temporal localization, J. Geophys. Res. Atmos., 120, 876 8783-8803, doi:10.1002/2015JD023363.

877 Fritts, D. C., P.-D. Pautet, K. Bossert, M. J. Taylor, B. P. Williams, H. Iimura, T. Yuan, N. J. 878 Mitchell, and G. Stober, 2014a: Quantifying gravity wave momentum fluxes with 879 Mesosphere Temperature Mappers and correlative instrumentation, J. Geophys. Res. Atmos., $880119,13,583-13,603$, doi:10.1002/ 2014JD022150.

881 Fritts, D. C., K. Wan, J. Werne, T. Lund, and J. H. Hecht, 2014c: Modeling Influences of Kelvin882 Helmholtz Instability Dynamics on Airglow, J. Geophys. Res., 119, 8858-8871, 883 doi:10.1002/2014JD021737.

884 Fritts, D. C., and P. K. Rastogi, 1985: Convective and dynamical instabilities due to gravity wave motions in the lower and middle atmosphere: Theory and observations, Radio Sci., 20, 12471277.

Fritts, D. C., S. A. Vadas, and Y. Yamada, 2002: An estimate of strong local gravity wave body forcing based on $\mathrm{OH}$ airglow and meteor radar observations, Geophys. Res. Lett., 29 (10), 10.1029/2001GL013753.

Fritts, D. C., and T. E. VanZandt, 1993: Spectral estimates of gravity wave energy and momentum fluxes, I: Energy dissipation, acceleration, and constraints, J. Atmos. Sci., 50, $3685-3694$.

Fritts, D. C., K. Wan, P. Franke, and T. Lund, 2012: Computation of clear-air radar backscatter from numerical simulations of turbulence, 3. Off-zenith measurements and biases throughout the lifecycle of a Kelvin-Helmholtz instability, J. Geophys. Res., 117, D17101, doi:10.1029/2011JD017179.

Fritts, D. C., and L. Wang, 2013: Gravity Wave - Fine Structure Interactions, Part 2: Energy 899 dissipation evolutions, statistics, and implications, J. Atmos. Sci., 70, 3735-3755, DOI:10.1175/JAS-D-13-059.1. 
900 Fritts, D. C., L. Wang, M. A. Geller, D. A. Lawrence, J. Werne, and B. B. Balsley, 2016:

901 Numerical Modeling of Multi-Scale Dynamics at a High Reynolds Number: Instabilities, 902 Turbulence, and an Assessment of Ozmidov and Thorpe Scales, J. Atmos. Sci., 73(2), 555903 578, doi: 10.1175/JAS-D-14-0343.1.

904 Fritts, D. C., L. Wang, and J. Werne, 2013: Gravity Wave - Fine Structure Interactions, Part 1:

905 Influences of fine structure form and orientation on flow evolution and instability, J. Atmos. 906 Sci., 70, 3710-3733, DOI:10.1175/JAS-D-13-055.1.

907 Fritts, D. C., L. Wang, J. Werne, T. Lund, and K. Wan, 2009a: Gravity wave instability 908 dynamics at high Reynolds numbers, 1: Wave field evolution at large amplitudes and high 909 frequencies, J. Atmos. Sci., 66, 1126-1148, doi:10.1175/2008JAS2726.1.

910 Fritts, D. C., L. Wang, J. Werne, T. Lund, and K. Wan, 2009b: Gravity wave instability 911 dynamics at high Reynolds numbers, 2: Turbulence evolution, structure, and anisotropy, $\underline{\mathbf{J}}$. $912 \quad$ Atmos. Sci., 66, 1149-1171, doi:10.1175/2008JAS2727.1.

913 Fritts, D. C., B. P. Williams, C.-Y. She, J. D. Vance, M. Rapp, F.-J. Lübken, F. J. Schmidlin, A. 914 Müllemann, and R. A. Goldberg, 2004: Observations of extreme temperature and wind 915 gradients near the summer mesopause during the MaCWAVE/MIDAS rocket campaign, $916 \quad$ Geophys. Res. Lett., 31, doi:10.1029/2003GL019389.

917 Fruman, M. D., Achatz, U., 2012: Secondary instabilities in breaking inertia-gravity waves., J. 918 Atmos. Sci., 69, 303-322.

919 Fruman, M. D., S. Remmler, U. Achatz, and S. Hickel, 2014: On the construction of a direct 920 numerical simulation of a breaking inertia-gravity wave in the upper mesosphere, J. 921 Geophys. Res. Atmos., 119, 11,613-11,640, doi:10.1002/2014JD022046.

922 Gargett, A. E., 1999: Velcro measurements of turbulent kinetic energy dissipation rate. J. Atmos. 923 Oceanic Technol., 16, 1973-1993.

924 Gossard, E. E., Gaynor, J. E., Zamora, R. J., and Neff, W. D., 1985: Fine structure of elevated 925 stable layers observed by sounder and in-situ tower sensors, J. Atmos. Sci., 42, 2156-2169. 
926 Hecht, J. H., 2004: Instability layers and airglow imaging, Rev. Geophys., 42, RG1001, 927 doi:10.1029/2003RG000131.

928 Hecht, J. H., A. Z. Liu, R. L. Walterscheid and R. J. Rudy, 2005: Maui Mesosphere and lower 929 thermosphere (Maui MALT) observations of the evolution of Kelvin-Helmholtz billows 930 formed near 86 km altitude, J. Geophys. Res., 110, D09S10, doi:10.1029/2003JD003908.

931 Hecht, J. H., A. Z. Liu, R. L. Bishop, J. H. Clemmons, C. S. Gardner, M. F. Larsen, R. G. Roble, 932 G. R. Swenson, and R. L. Walterscheid. 2004: An overview of observations of unstable 933 layers during the Turbulent Oxygen Mixing Experiment (TOMEX), J. Geophys. Res., 109, 934 D2, DOI: 10.1029/2002JD003123.

935 Hecht, J. H., K. Wan, L. J. Gelinas, D. C. Fritts, R. L. Walterscheid, S. J. Franke, G. R. Swenson, 936 F. A. Vargas, A. Z. Liu, M. J. Taylor, and P.D. Pautet, 2014: The lifecycle of instability 937 features measured from the Andes Lidar Observatory over Cerro Pachon on March 24, 2012, $938 \quad$ J. Geophys. Res., 119, 8872-8898, doi:10.1002/2014JD021726.

939 Lawrence, D. A., and B. B. Balsley, 2013: High-Resolution Atmospheric Sensing of Multiple 940 Atmospheric Variables using the DataHawk Small Airborne Measurement System, J. Atmos. 941 And Ocean. Tech., Vol. 30, 2352-2366, doi: http://dx.doi.org/10.1175/JTECH-D-12-00089.1 942 Lelong, M.-P., and T. J. Dunkerton, 1998a: Inertia-gravity wave breaking in three dimensions. 943 Part I: Convectively stable waves. J. Atmos. Sci., 55, 2473-2488.

944 Lelong, M.-P., and T. J. Dunkerton, 1998b: Inertia-gravity wave breaking in three dimensions.

945 Part II: Convectively unstable waves. J. Atmos. Sci., 55, 2489-2501.

946 Lehmacher, G. A., L. Guo, E. Kudeki, P. M. Reyes, A. Akgiray, and J. L. Chau, 2007: High947 resolution observations of mesospheric layers with the Jicamarca VHF radar, Adv. Space 948 Res., 40(6), 734-743.

949 Lehmacher, G. A., T. D. Scott, M. F. Larsen, S. G. Bilen, C. L. Croskey, J. D. Mitchell, M. 950 Rapp, F.-J. Lübken, and R. L. Collins, 2011: The Turbopause experiment: Atmospheric 951 stability and turbulent structure spanning the turbopause altitude, Ann. Geophys., 29, 2327952 2339, doi:10.5194/angeo-29-2327-2011. 
953 Lilly, D. K., 1983: Stratified turbulence and the mesoscale variability of the atmosphere, J.

$954 \quad$ Atmos. Sci., 40, 749-761.

955 Lübken, F.-J., 1997: Seasonal variation of turbulent energy dissipation rates at high latitudes as 956 determined by in situ measurements of neutral density fluctuations, J. Geophys. Res., 102, $957 \quad \mathrm{D} 12,13,441-13,456$.

958 Lübken, F.-J., M. Rapp, and P. Hoffmann, 2002: Neutral air turbulence and temperatures in the 959 vicinity of polar mesosphere summer echoes, J. Geophys. Res., 107, 4273, 960 doi:10.1029/2001JD000915.

961 Luce, H., M. Crochet, F. Dalaudier, and C. Sidi, 1995: Interpretation of VHF ST radar vertical 962 echoes from in situ temperature sheet observations. Radio Sci., 30, 1002-1025.

963 Luce, H., Crochet, M., Hanuise, C., Yamamoto, M., and Fukao, S., On the interpretation of the 964 layered structures detected by MST radars in dual Frequency Domain Interferometry (FDI) 965 mode, Radio Sci., 34, 1077-1083, 1999.

966 Luce, H., G. Hassenpflug, M. Yamamoto, and S. Fukao, 2006: High-resolution vertical imaging 967 of the troposphere and loer stratosphere using the new MU radar system, Ann. Geophys., 24, 968 791-805, www.ann-geophys.net/24/791/2006.

969 McIntyre, M. E., 1989: On dynamics and transport near the polar mesopause in summer, J. $970 \quad$ Geophys. Res., 94(D12), 14,617-14,628.

971 Moum, J. N., 1996: Energy-containing scales of turbulence in the ocean thermocline. J. Geophys. $972 \quad$ Res., 101, 14 095-14 101.

973 Muschinski, A., and C. Wode, 1998: First in situ evidence for coexisting submeter temperature 974 and humidity sheets in the lower free troposphere. J. Atmos. Sci., 55, 2893-2906.

975 Nastrom, G. D., and F. D. Eaton, 2001: Persistent layers of enhanced Cn2 in the lower 976 stratosphere from radar. Radio Sci., 36, 137-149.

977 Osborn, T. R., 1980: Estimates of the local rate of vertical diffusion from dissipation 978 measurements. J. Phys. Oceanogr., 10, 83-89. 
979 Osborn, T. R., and Cox, 1972: Oceanic fine structure, Geophys. Fluid Dyn., 3(1), 321-345, $980 \quad$ DOI: $10.1080 / 03091927208236085$.

981 Pfrommer, T., P. Hickson, and C.-Y. She, 2009: A large-aperture sodium fluorescence lidar with 982 very high resolution for mesopause dynamics and adaptive optics studies, Geophys. Res. 983 Lett., 35(15), L15831, DOI: 10.1029/2009GL038802.

984 Rapp, M., B. Strelnikov, A. Müllemann, F.-J. Lübken, and D. C. Fritts, 2004: Turbulence 985 measurements implications for gravity wave dissipation during the MaCWAVE/MIDAS 986 summer rocket program, Geophys. Res. Lett., 31, doi:10.1029/2003GL019325.

987 Remmler, S., Fruman, M. D., Hickel, S., 2013: Direct numerical simulation of a breaking inertia988 gravity wave., J. Fluid Mech., 722, 424-436.

989 Sato, T., and R. F. Woodman, 1982: Fine altitude resolution radar observations of stratospheric 990 turbulent layers by the Arecibo $430 \mathrm{MHz}$ radar, J. Atmos. Sci., 39, 2546-2552.

991 Schneider, A., M. Gerding, and F.-J. Lübken, 2014: Comparing turbulent parameters obtained 992 from LITOS and radiosonde measurements, Atmos. Chem. Phys. Discussion, 14, 19033993 19053, doi:10.5194/acpd-14-19033-2014.

994 Smyth, W. D., and J. N. Moum, 2000: Length scales of turbulence in stably stratified mixing 995 layers. Phys. Fluids, 12, 1327-1342.

996 Strelnikov, B., M. Rapp, I. Strelnikova, N. Engler, and R. Latteck, 2009: Small-scale structures 997 in neutrals and charged aerosol particles as observed during the ECOMA/MASS rocket 998 campaign, Ann. Geophys., 27, 1449-1456, www.ann-geophys.net/27/1449/2009.

999 Sutherland, B. R., 2001: Finite-amplitude internal wavepacket dispersion and breaking, J. Fluid $1000 \quad$ Mech, 429, 343-380.

1001 Sutherland, B. R., 2006b: Weakly nonlinear internal gravity wavepackets, J. Fluid Mech., 569, $1002 \quad 249-258$.

1003 Sutherland, B. R., 2006a: Internal wave instability: Wave-wave versus wave-induced mean flow 1004 interactions, Phys. Fluids 18, 074107, doi:10.1063/1.2219102. 
1005 Thorpe, S. A., 1977: Turbulence and mixing in a Scottish loch, Philos. Trans. Roy. Soc. Lon., $1006 \quad$ A286, 125-181.

1007 Thorpe, S. A., 1999: On the breaking of internal waves in the ocean, J. Phys. Ocean., 29, 2433$1008 \quad 2441$

1009 Thorpe, S. A., 2005: The Turbulent Ocean, Cambridge Univ. Press, Cambridge, U. K.

1010 Walterscheid, R. L., 1981: Inertio-gravity wave induced accelerations of mean flow having an 1011 imposed periodic component: Implications for tidal observations in the meteor region, J. 1012 Geophys. Res., 86, 9698-9706.

1013 Werne, J., and D. C. Fritts, 1999: Stratified shear turbulence: Evolution and statistics, Geophys.

$1014 \quad$ Res. Lett., 26, 439-442.

1015 Wesson, J. C., and M. C. Gregg, 1994: Mixing in the Camarinal Sill in the Strait of Gibraltar. J. 1016 Geophys. Res., 99, 9847-9878.

1017 Witt G., 1962: Height, structure and displacements of noctilucent clouds, Tellus 14, 1-18.

1018 Woodman, R. F., and A. Guillen, 1974: Radar Observations of Winds and Turbulence in the

1019 Stratosphere and Mesosphere. Journal of the Atmospheric Sciences, 31, 493-505, doi: $1020 \quad 10.1175 / 1520-0469(1974) 031<0493:$ ROOWAT $>2.0 . C O ; 2$.

1021 Woods, J. D. and R. L. Wiley, 1972: Billow turbulence and ocean microstructure, Deep Sea Res., $1022 \quad 19,87-121$.

1023 Yamada, Y., H. Fukunishi, T. Nakamura, and T. Tsuda, 2001: Breakdown of small-scale quasi1024 stationary gravity wave and transition to turbulence observed in $\mathrm{OH}$ airglow, Geophys. Res. 1025 Lett., 28, 2153-2156.

1026

1027

1028

1029

1030

1031 



1035 Figure 1. Tilted computational domains aligned along the primary GW for (a) the idealized DNS

1036 of GW breaking for $a=0.9$ and 1.1 and (b) the MSD DNS of a superposed initial GW and mean 1037 small-scale shear flow. The domain and geophysical coordinates are $\left(x^{\prime}, y^{\prime}, z^{\prime}\right)$ and $(x, y, z)$ in each 1038 case and blue and red arrows and oscillatory curves show the initial GW and mean shear flows. 1039 See text for additional details on other initial conditions and flow parameters. 



1042 Figure 2. Measurements of PMC layer thickness with the Rayleigh-Mie-Raman lidar at 1043 ALOMAR from (a) 23:30-00:30 UT on 30 June to 1 July 2013 and (b) 00:00-00:12 UT on 1 July 1044 2013. The times and altitudes shown in (b) are highlighted in the yellow rectangle in (a). Note 1045 that the layer FWHM can often be $<100 \mathrm{~m}$.

1046 

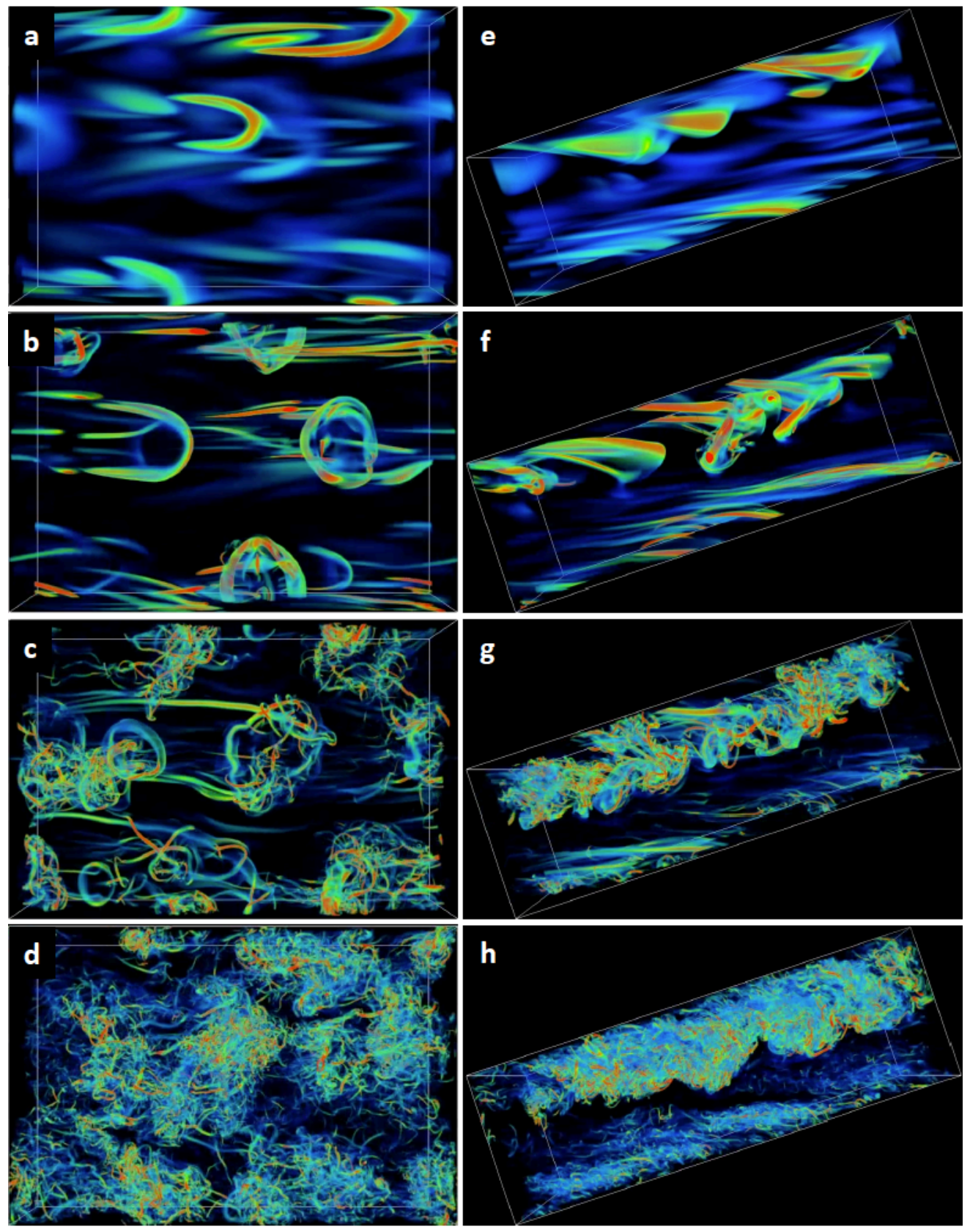

1053 Figure 3. 3D volumetric views of $\lambda_{2}$ from (a-d) below and (e-h) the side showing the evolution 1054 of initial instabilities and turbulence accompanying the GW breaking DNS with $a=0.9$ at 21,22 , 105523 , and $24 T_{b}$ (top to bottom). The GW is shown propagating upward and to the right for more 1056 convenient comparisons with the PMC imaging from the ground. The color scale varies from 1057 weak (light blue) to intense (yellow/red) rotation. 

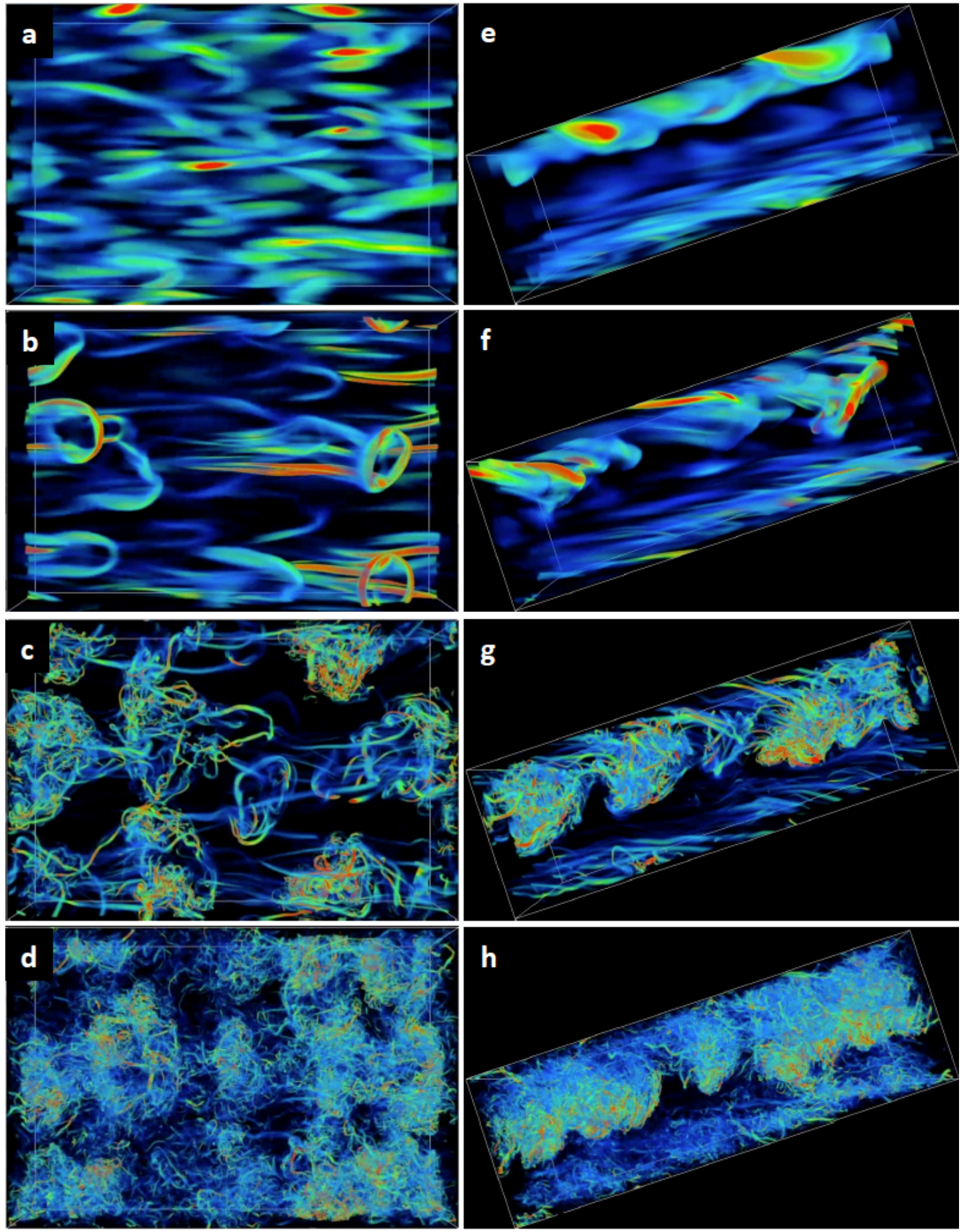

1059 Figure 4. As in Fig. 3 for the GW breaking DNS with $a=1.1$ at 9.2, 10.2, 11.2, and $12.2 T_{b}$.

1060 

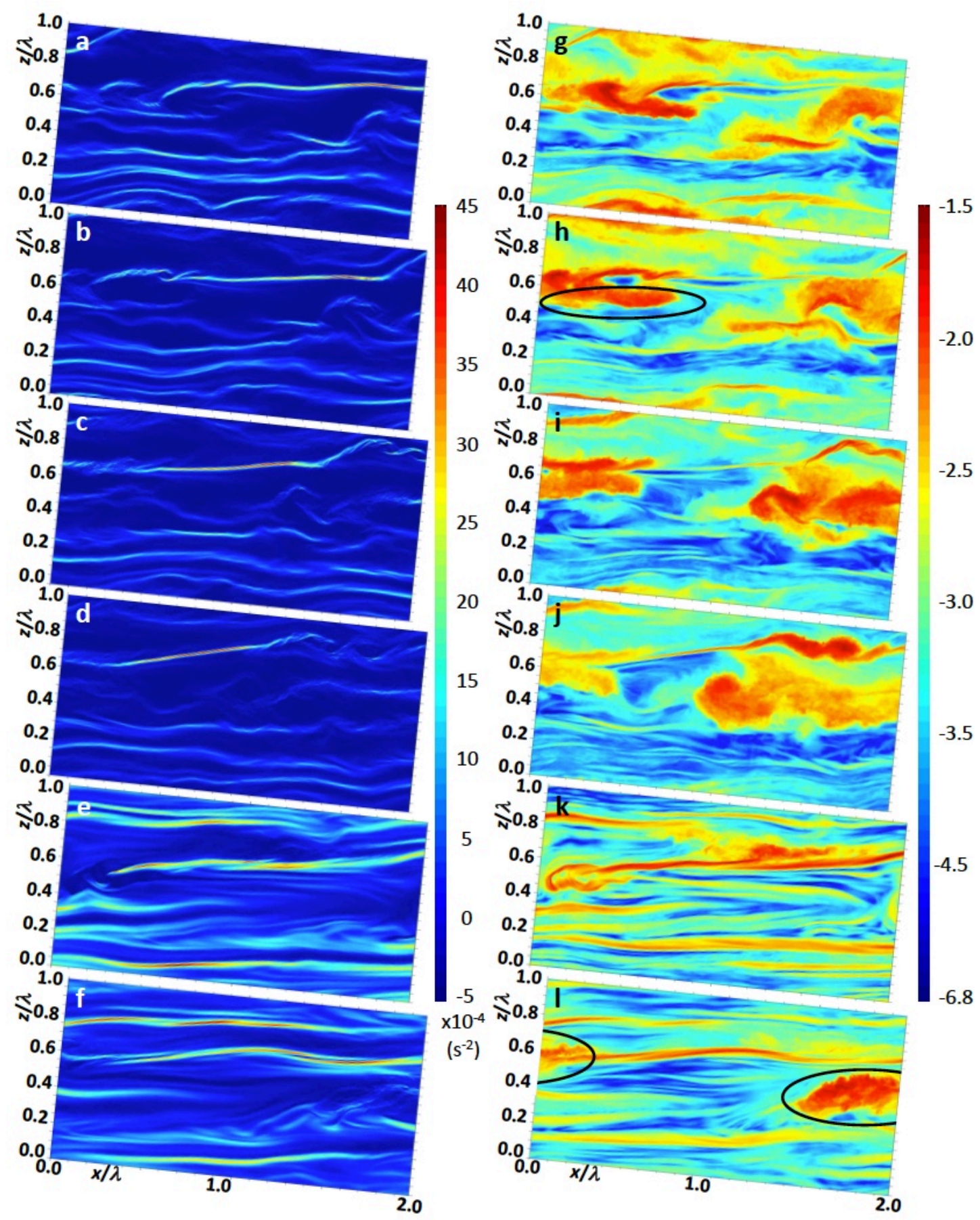

1063 Figure 5. Spanwise-averaged $N^{2}(x, z)$ and $\log _{10} \varepsilon(x, z)$ for the MSD DNS described in Section 4.2 1064 showing an evolving GW breaking and KHI at 10, 10.4, 11, and $11.6 T_{b}$ (a-d and g-j) and an 1065 intrusion event at 31.7 and $33 T_{b}$ (e and f, $\mathrm{k}$ and 1 ). The $N^{2}$ color scale has a maximum of $\sim 11 N_{0}^{2}$ 1066 and the $\log _{10} \varepsilon(x, z)$ color scale is nondimensional and must be scaled by $S=\lambda_{z}^{2} / T_{b}^{3}$. 



1069 Figure 6. Ground-based wide-FOV PMC images viewing NNE from Trondheim, Norway on 1 1070 August 2009 at 23:20, 23:30, 23:40, and 23:50 UT (a-d). The images are projected to the PMC 1071 altitude of $\sim 82 \mathrm{~km}$ viewed from above, with $\mathrm{N}$ and $\mathrm{E}$ up and to the right. The FOV spans $1072>200 \times 200 \mathrm{~km}$, yellow circles highlight the GW instability event of interest, the green trapezoid 1073 in (c) indicates the narrower FOV employed to examine the GW instability structures, and the 1074 lines and corresponding arrows show the phase orientations and propagation directions of the 1075 primary GWs. 




1078 Figure 7. Narrow FOV images of the GW breaking event showing the evolutions of vortex rings 1079 from 23:42-23:45 UT (a-d) at a slant angle of $\sim 21^{\circ}$ below the horizontal and ahead and to the 1080 right of the GW propagation direction (towards the SE). Yellow ovals highlight the major vortex 1081 rings occurring during this phase of the event; yellow arrows show the motion of the trailing 1082 vortices for the most upstream (eastward vortex ring). Panels (e) and (f) show the projected fields 1083 in (a) and (d). 
1085
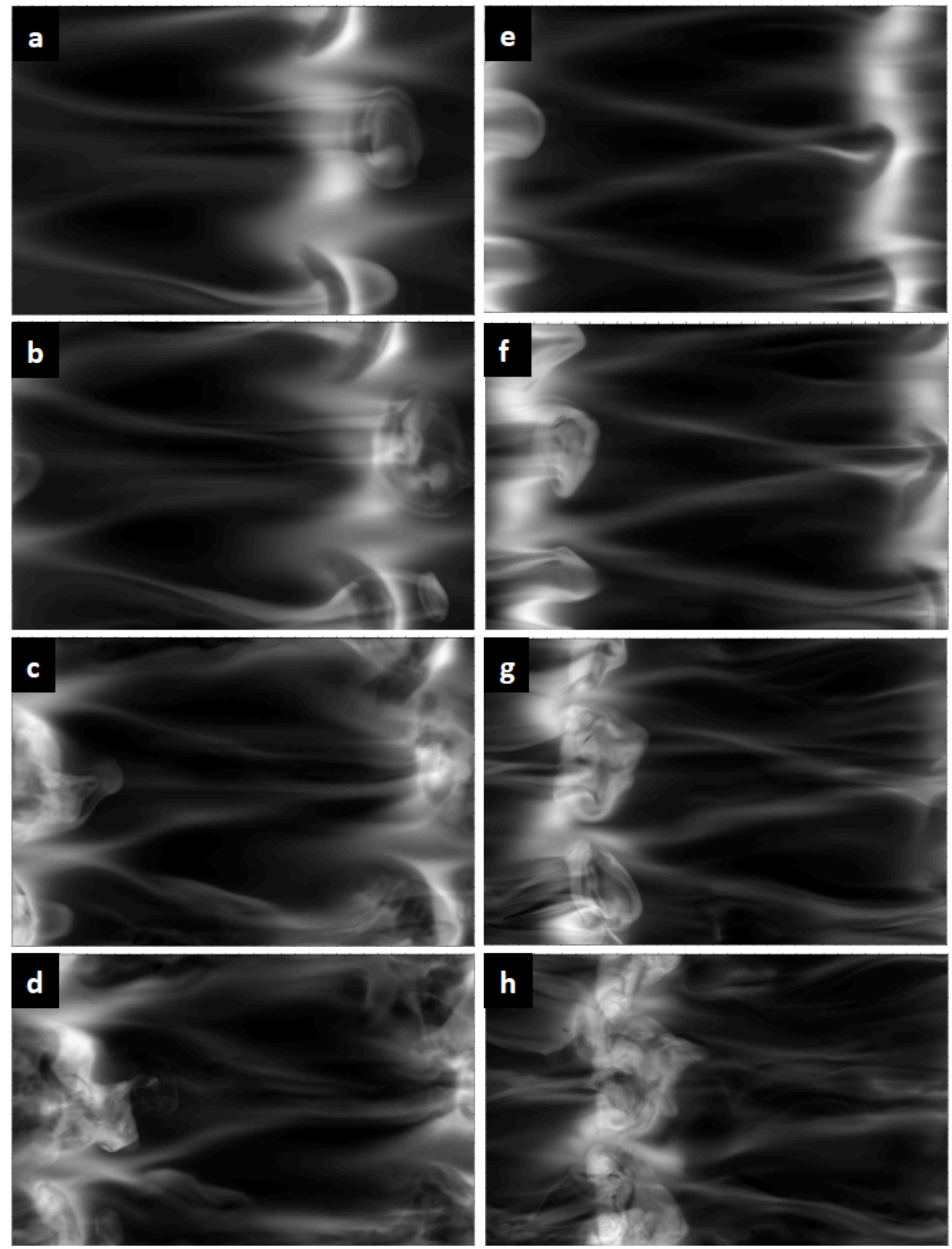

1086 Figure 8. Simulated PMC responses in the GW breaking DNS for $a=0.9$ from $22-23$ (a-d) $T_{b}$ and 1087 for $a=1.1$ from 10.2-11.2 (e-h) at $\sim 0.33 T_{b}$ intervals having a PMC layer centered on the vortex 1088 rings in the most unstable phase of the $\mathrm{GW}$ with $z_{F W H M}=0.1 \lambda_{z}$ in each case. 


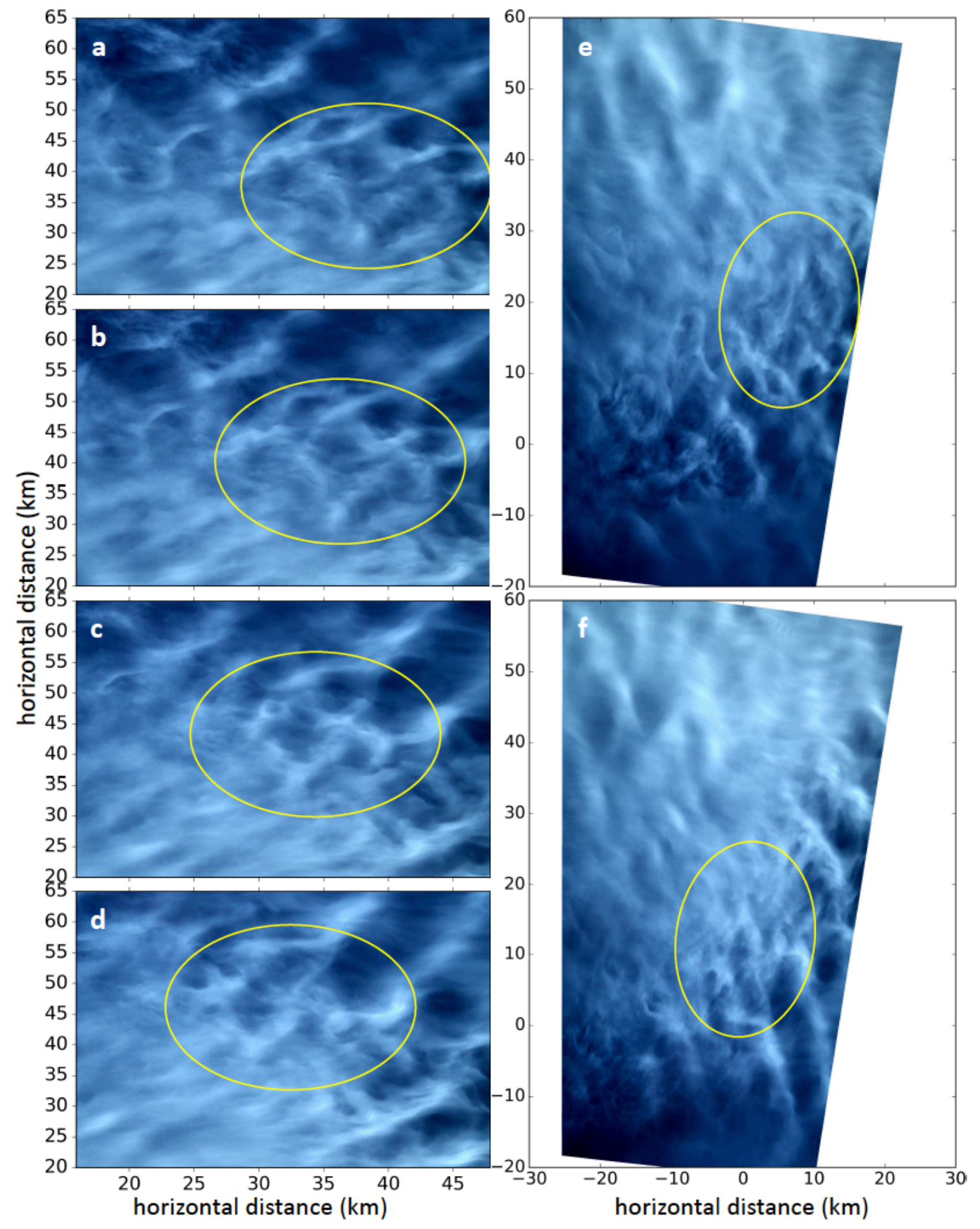

1089 Figure 9. As in Fig. 7 for 23:47-23:50 UT. The yellow ovals in this case show new vortex rings 1090 that formed to the SE of those shown in Fig. 7. 

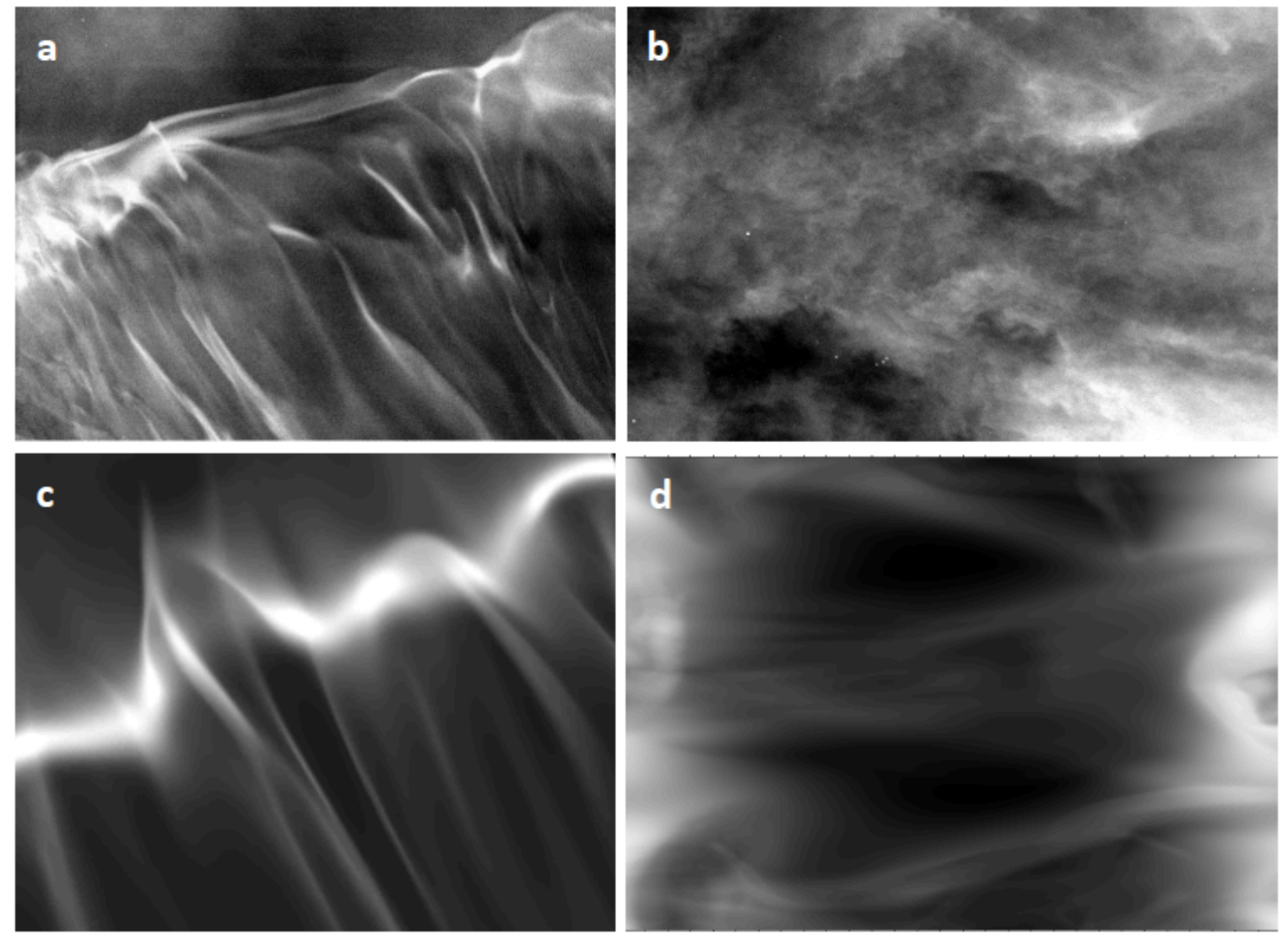

1094 Figure 10. PMC imaging by the EBEX star cameras showing a GW breaking front (a) and a zig1095 zag pattern at the time of initial streamwise vortex generation (b) in projected FOVs of 4.4x3.9 $1096 \mathrm{~km}$. Panels (c) and (d) show corresponding images of simulated PMCs with $z_{F W H M}=100 \mathrm{~m}$ from 1097 the GW breaking DNS shown in Fig. 4 at $10.25 T_{b}$ and Fig. 3 at $\sim 22 T_{b}$, respectively. 



1107 Figure 11. As in Fig. 10 showing PMC images of vortex rings at various scales, orientations, and 1108 evolution stages (a-c). PMC image FOVs are 4.4x3.9, 4x3.9, and 4.4x2 km, respectively. Panels 1109 (d) and (e) show two images of vortex rings from the GW breaking DNS for $a=1.1$ at $\sim 10.5 T_{b}$ 1110 with $z_{F W H M}=0.2 \lambda_{z}$ at the level of the vortex rings (d) and with $z_{F W H M}=0.05 \lambda_{z}$ somewhat above. 



1114 Figure 12. As in Fig. 10 showing a PMC image of cusp-like structures below an apparent region 1115 of local GW breaking in an MSD environment (a) and a simulation of similar features from a 1116 DNS of an MSD flow (b). The projected FOV of the PMC image in (a) is $4.4 \times 3.9 \mathrm{~km}$. The MSD 1117 dynamics occur in the black oval in Fig. 5h. Arrows in each panel show the direction of shearing 1118 below these structures. 

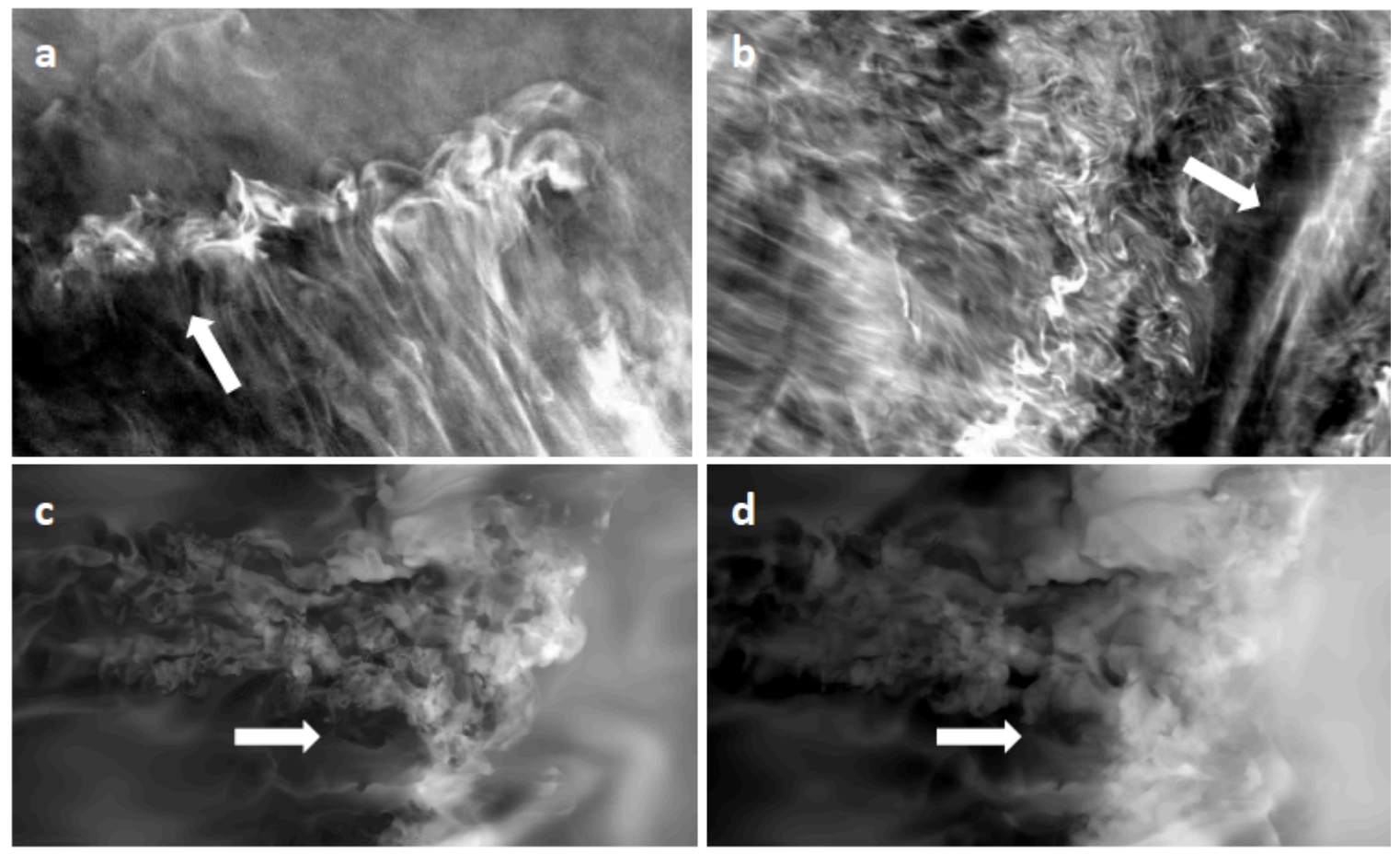

1120 Figure 13. As in Fig. 12 showing PMC images of apparent intrusions (a and b) in projected 1121 FOVs of 3.7x2.6 and 4.4x3.9 km, respectively. Panels (c) and (d) show a simulated PMC arising 1122 from the intrusion in the black oval in Fig. 5h with $z_{F W H M}=100$ and $300 \mathrm{~m}$ (c and d) to illustrate 1123 the increased sensitivity to small-scale features by a thinner PMC layer. 

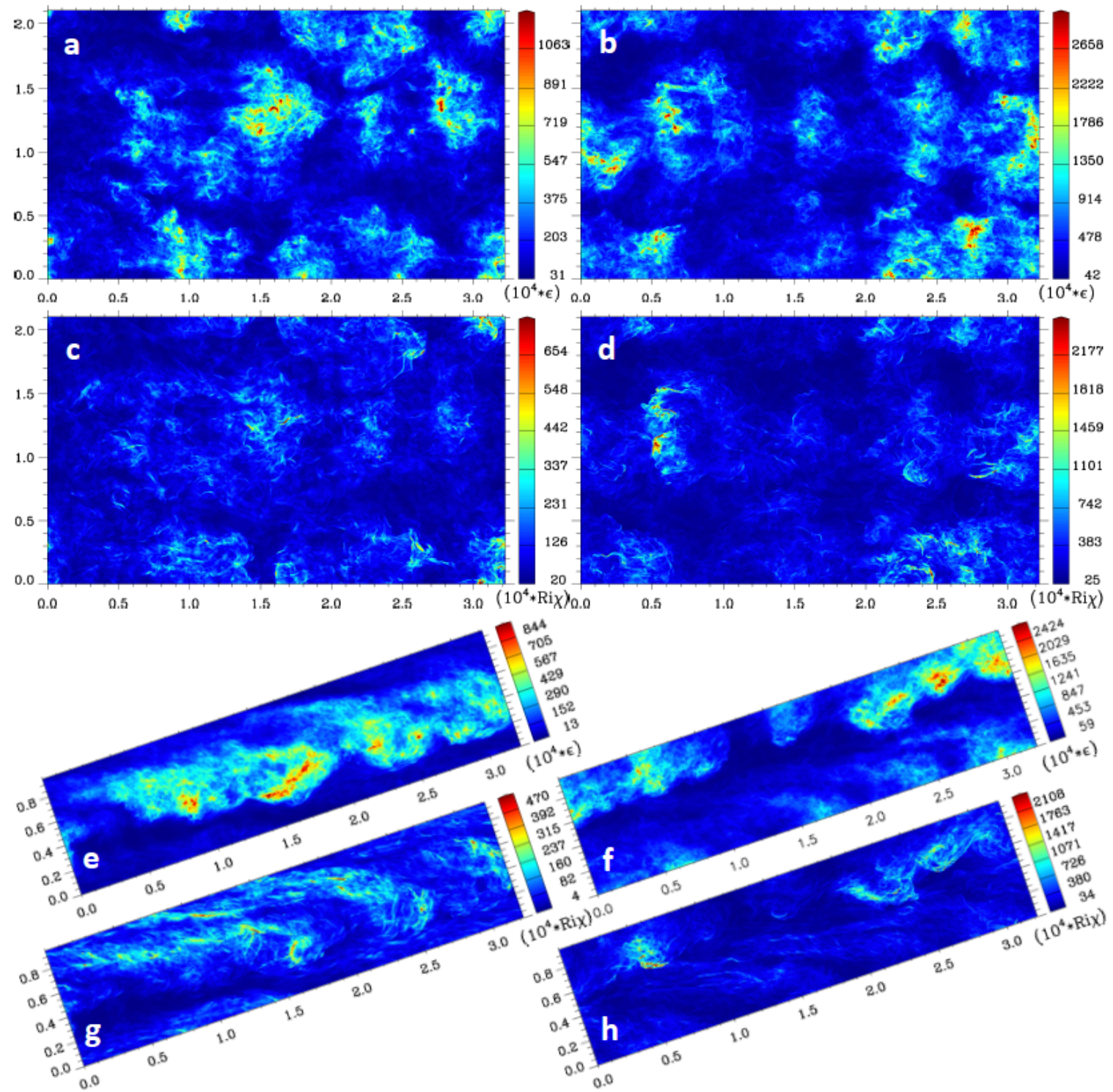

1135 Figure 14. Horizontal (top panels) and vertical (bottom panels) cross sections of vertically- and

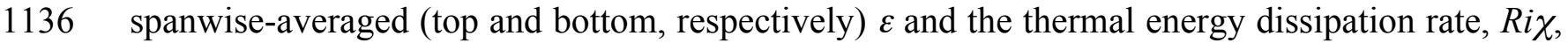

1137 for the DNS of GW breaking shown in Figs. 3 and 4. $\varepsilon$ cross sections are shown in panels (a) and

1138 (e) for $a=0.9$ and in panels (b) and (f) for $a=1.1$. Corresponding Ri $\chi$ cross sections are shown in

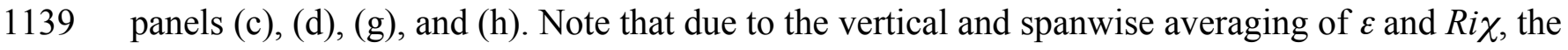

1140 ranges of values in the color scales are now $\sim 2$ rather than $\sim 4$ decades in each case. The color





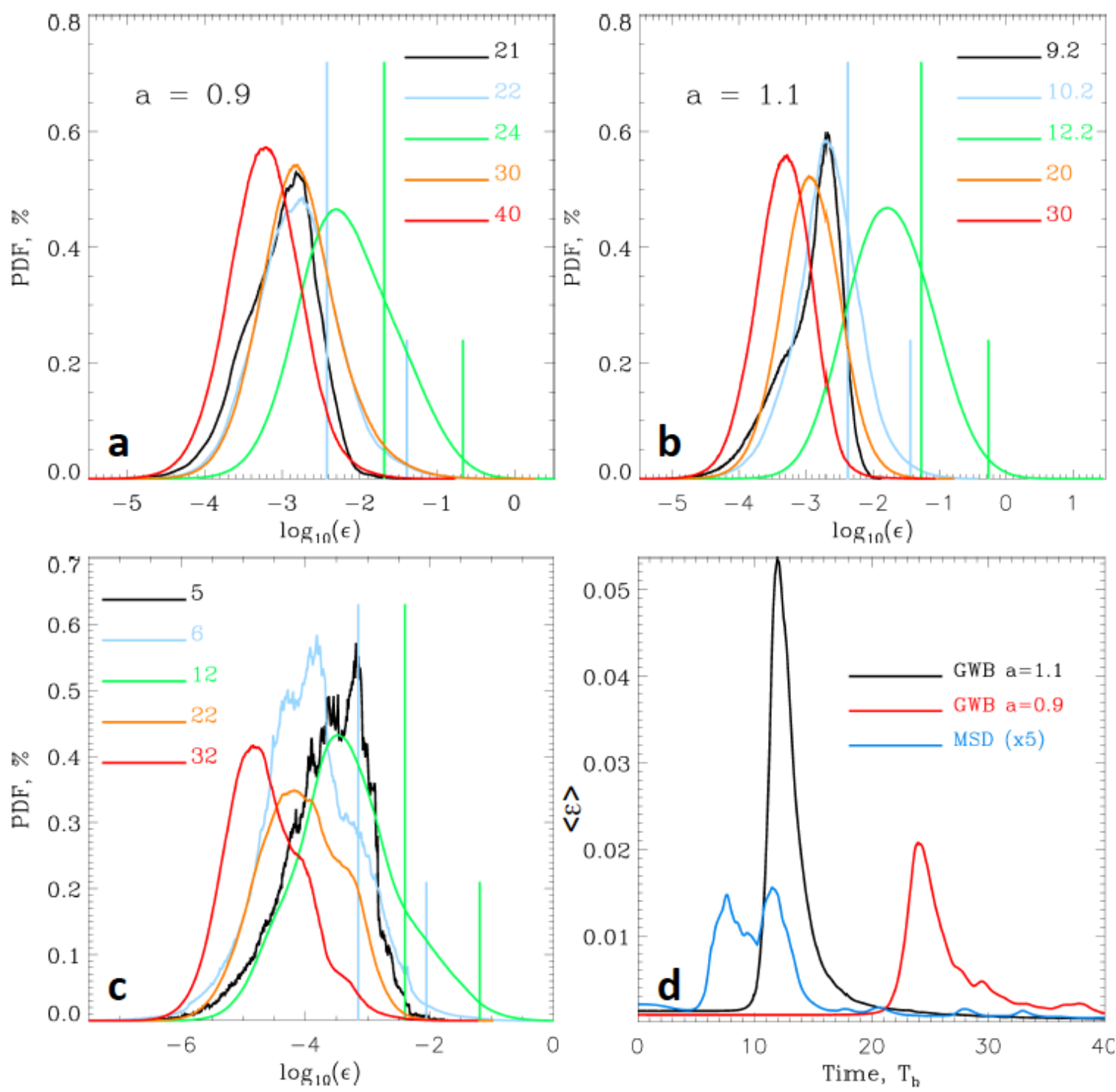

1147 Figure 15. PDFs of $\log _{10} \varepsilon$ for GW breaking with $a=0.9$ and $a=1.1$, and for the MSD DNS are

1148 shown in (a-c), respectively. Times shown in each panel extend from initial instability stages to

1149 late in the turbulence decay. Vertical lines for the second and third times in each case show $<\varepsilon>$

1150 and $\varepsilon_{\text {high. }}$. Nondimensional domain-mean $\varepsilon$ are shown from $0-40 T_{b}$ for the three DNS in panel

1151 (d). Note that turbulence intensities are very much smaller for the MSD than for the GW 1152 breaking cases for the same domain depths. 
1158
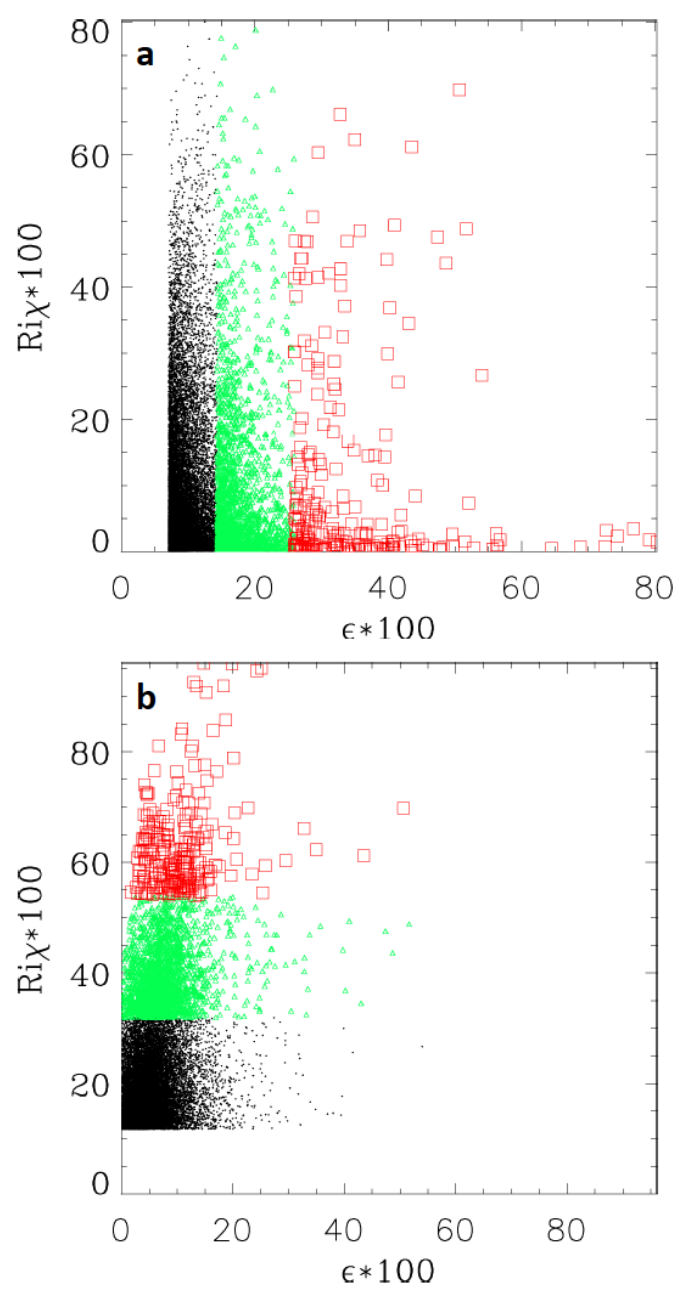





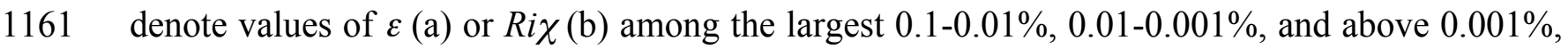
1162 respectively.

1163 